OPEN ACCESS

Edited by:

Fabio Minervini,

Università degli Studi di Bari, Italy

Reviewed by:

Maurizio Ciani,

Università Politecnica delle Marche,

Pasquale Russo,

University of Foggia, Italy

Thibault Nidelet,

INRA - Montpellier Centre, France

${ }^{*}$ Correspondence:

Luc De Vuyst

luc.de.vuyst@vub.be

Specialty section:

This article was submitted to

Food Microbiology,

a section of the journal

Frontiers in Microbiology

Received: 10 September 2018

Accepted: 29 October 2018

Published: 19 November 2018

Citation:

De Roos J, Vandamme $P$ and

De Vuyst L (2018) Wort Substrate

Consumption and Metabolite

Production During Lambic Beer

Fermentation and Maturation Explain the Successive Growth of Specific

Bacterial and Yeast Species.

Front. Microbiol. 9:2763.

doi: 10.3389/fmicb.2018.02763

\section{Wort Substrate Consumption and Metabolite Production During Lambic Beer Fermentation and Maturation Explain the Successive Growth of Specific Bacterial and Yeast Species}

\author{
Jonas De Roos ${ }^{1}$, Peter Vandamme ${ }^{2}$ and Luc De Vuyst ${ }^{\text {* }}$ \\ ${ }^{1}$ Research Group of Industrial Microbiology and Food Biotechnology, Bioengineering Sciences Department, Vrije Universiteit \\ Brussel, Brussels, Belgium, ${ }^{2}$ Laboratory for Microbiology, Department of Biochemistry and Microbiology, Ghent University, \\ Ghent, Belgium
}

The present study combined high-throughput culture-dependent plating and cultureindependent amplicon sequencing with a metabolite target analysis to systematically dissect the identity, evolution, and role of the microorganisms, substrates, and metabolites during the four-phase fermentation and maturation process of lambic beer production. This led to the following new insights. The changing physicochemical parameters and substrate and metabolite compositions of the fermenting wort and maturing lambic beer provoked several transitions between microbial species and explained the four-step production process. Manual wort acidification with lactic acid shortened the enterobacterial phase and thus kept biogenic amine formation by enterobacteria present during the early stages of fermentation at a minimum. Growth advantages during the alcoholic fermentation phase caused a transition from the prevalence by Hanseniaspora uvarum and Kazachstania species to that by Saccharomyces cerevisiae and later on Saccharomyces kudriavzevii, due to changing environmental parameters. During the acidification phase, Pediococcus damnosus was prevalent and performed a malolactic fermentation. Acetobacter pasteurianus produced acetic acid and acetoin. Upon maturation, Dekkera species appeared, together with $P$. damnosus and Pichia membranifaciens, thereby contributing to acetic acid production, depending on the oxygen availability. Moreover, the Dekkera species consumed the acetoin produced by the acetic acid bacteria for redox balancing. The breakdown of maltooligosaccharides seemed to be independent of the occurrence of Dekkera species and started already early in the fermentation process.

Keywords: lambic beer, Dekkera, malolactic fermentation, MALDI-TOF MS, amplicon sequencing

\section{INTRODUCTION}

Worldwide, the beer market primarily consists of lager beers produced through bottom fermentation of barley malt, water, and hop with the brewers' yeasts Saccharomyces bayanus or Saccharomyces pastorianus (Bokulich and Bamforth, 2013, 2017; Howard, 2014). However, top-fermented ale beers now know high production volumes as well, either on a 
global scale or regionally. Regional production often takes place according to traditional recipes that make use of a variety of ingredients (e.g., diverse cereals, herbs, and spices). Top-fermented beer production is carried out mainly with Saccharomyces cerevisiae (Briggs et al., 2004). In contrast, autochthonous microorganisms [spontaneously growing yeasts and lactic acid bacteria (LAB)] or uncharacterized mixed starter cultures (yeast-LAB starter cultures maintained inhouse) perform the fermentation and/or maturation processes of certain traditional Belgian acidic ales (Verachtert and Iserentant, 1995; Martens et al., 1997; Verachtert and Derdelinckx, 2005; Snauwaert et al., 2016). Belgian lambic beers are a typical example of spontaneously fermented acidic beers, which are produced in specialized breweries (Spitaels et al., 2014, 2015, 2017). Traditional acidic beers are currently attracting increasing attention worldwide (Pothakos et al., 2016; Spitaels et al., 2017; De Roos and De Vuyst, 2018). American craft brewers for example have adopted the production methods of Belgianstyle acidic ales to produce spontaneously fermented American coolship ales (ACAs; Bokulich et al., 2012).

Acidic lambic beers are obtained through spontaneous fermentation of barley malt, unmalted wheat, and aged dry hops (De Keersmaecker, 1996; Pothakos et al., 2016; Spitaels et al., 2017). The spontaneous fermentation and maturation process can proceed up to 3 years and is traditionally carried out in wooden casks (Verachtert and Iserentant, 1995). Most knowledge about these beers originates from microbiological studies performed several decades ago. These studies relied entirely on culture-dependent microbiological analyses and a restricted metabolite target analysis with techniques that are outdated now (Van Oevelen et al., 1976, 1977; Spaepen et al., 1978, 1979; Verachtert, 1983; Verachtert and Dawoud, 1984; Verachtert et al., 1989; Martens et al., 1991, 1992; Shanta Kumara and Verachtert, 1991; Verachtert and Iserentant, 1995). Most of these studies have shown that four phases can be distinguished during a traditional lambic beer production process: (i) the enterobacterial and wild (oxidative) yeast phase from the start up to 1 month of fermentation, which is characterized by low concentrations of organic acids and ethanol; (ii) the main fermentation phase (from month 1 until month 4) dominated by the yeast species $S$. cerevisiae and S. pastorianus, which is characterized by the production of ethanol and carbon dioxide; (iii) the acidification phase (from month 4 until month 10) dominated by the LAB species Pediococcus damnosus and/or Lactobacillus brevis, which is characterized by the production of high concentrations of lactic acid; and (iv) the maturation phase (after 10 months) dominated by the yeast species Dekkera bruxellensis and the LAB species $P$. damnosus and/or L. brevis, which is characterized by the production of ethyl acetate and ethyl lactate (Verachtert and Iserentant, 1995; Verachtert and Derdelinckx, 2005).

Recently, lambic beer production processes performed in a traditional and common brewery were revisited through a followup for 24 and 12 months, respectively, applying primarily upto-date culture-dependent microbiological analysis on samples taken as a function of time but without monitoring substrate consumption and metabolite production (Spitaels et al., 2014, 2015). These studies have shown the same succession of microbial species mentioned above, albeit that manual acidification of the wort with lactic acid before the start of the fermentation at the common brewery, which is an adapted practice in most today's lambic breweries too, eliminates the first enterobacterial phase and results in an overlap of the acidification and maturation phases. Acidification would avoid biogenic amine production by enterobacteria (Spitaels et al., 2015). Yet, the effect on the total biogenic amine contents of these beers is unclear, as not only enterobacteria but also other lambic beer inhabitants such as LAB species and D. bruxellensis are able to produce these compounds (Izquierdo-Pulido et al., 1995, 1996; Kalac and Krizek, 2003; Oelofse et al., 2008; Spano et al., 2010). Further, it has been shown that acetic acid bacteria (AAB) are present abundantly during major periods of the first fermentation year of traditional lambic beer production and produce significantly higher concentrations of acetic acid and acetoin at the liquid/air interphase of the casks, due to higher $\mathrm{AAB}$ counts and metabolic activity compared to the middle and bottom of the fermenting wort and maturing beer in the casks (De Roos et al., 2018). ACA production closely parallels the microbial succession seen during lambic beer production. Minor differences between both beer productions occur in the enterobacterial species present and the abundant presence of $S$. cerevisiae from the start of ACA production, the latter maybe due to the production of ACAs in a common brewery. Further, aerobic bacteria, including $\mathrm{AAB}$, become more prevalent after 1 year of ACA production, although Lactobacillales remain dominant (Bokulich et al., 2012). This contrasts with certain lambic beer production processes, in which $\mathrm{AAB}$ do occur until 24 months of maturation, but are most prevalent after 3 months of fermentation (De Roos et al., 2018). Hence, it is not yet clear how shaping of the microbial communities during lambic beer fermentation and maturation occurs.

The present study aimed at a systematic dissection of the identity, evolution, and role of the microorganisms, substrates, and metabolites in oak casks used for the spontaneous fermentation and maturation process of Belgian, traditional lambic beer. Therefore, high-throughput culture-dependent and culture-independent microbiological techniques, in combination with a metabolite target analysis of fermentationand maturation-related compounds, were performed during a production period of 24 months.

\section{MATERIALS AND METHODS}

\section{Lambic Beer Production and Sampling}

Two lambic beer production processes were sampled in a traditional lambic brewery located in the Senne river valley southwest of Brussels (Belgium) in November 2014; these were the same processes, for which the $\mathrm{AAB}$ communities were studied, as reported before (De Roos et al., 2018). Briefly, wort of $12.6^{\circ} \mathrm{P}$ was prepared according to the brewer's recipe, manually acidified with lactic acid after wort boiling, and cooled overnight in a coolship open to the environmental air. The wort was then 
chilled to $4^{\circ} \mathrm{C}$ and transferred by means of appropriate tubings into oak casks of 6601 for fermentation and maturation. These casks originated from port wine production but were already used several times to produce lambic beer. Before use, the casks were cleaned with high-pressurized water and fumigated with sulfur sticks to inhibit mold growth after cleaning. Before filling, the casks were opened to the environmental air to remove the remaining sulfur vapors. Two identical casks (further referred to as casks 1 and 2), filled with wort of the brew mentioned above and located next to each other in the bottom row of tens of casks in a cellar (ambient temperature ranging from 9 to $20^{\circ} \mathrm{C}$, due to seasonal effects), were sampled as a function of time, representing biological duplicates.

A sample of $100 \mathrm{ml}$ was taken from the cooled wort before its transfer to the wooden casks. Further samples (100 ml) were taken $1 \mathrm{~h} ; 1$ and 3 days; 1, 2, 3, and 7 weeks; and 3, 6, 7.5 (only for LAB in cask 2), 9, 13, 18, and 24 months after its transfer. A volumetric adjustment of the liquid level in the casks was only performed at the timepoint of 13 months Aseptic sampling was performed at different heights of the fermenting wort and maturing beer in the casks (top, middle, and bottom) by applying flame sterilization and making use of $\gamma$-irradiated jumbo pipets $(900 \mathrm{~mm}$, highdensity polyethylene; VWR International, Darmstadt, Germany) through the cork-plugged bunghole of the casks $(50 \mathrm{ml}$ per insertion). Samples were put on ice before their transfer to the laboratory for analysis. Part of the samples was analyzed immediately (physicochemical parameters and microbiological plating); another part $(50 \mathrm{ml})$ was centrifuged $(4618 \times g$, $20 \mathrm{~min}, 4^{\circ} \mathrm{C}$ ) to obtain cell pellets and cell-free supernatants that were stored at $-20^{\circ} \mathrm{C}$ for microbiological and metabolite target analysis, respectively.

\section{Determination of Physicochemical Parameters}

Immediately after sampling, the $\mathrm{pH}$, temperature, and apparent attenuation were measured on site. The $\mathrm{pH}$ was determined with a portable $\mathrm{pH}$ meter ( $\mathrm{pH} 10$ Pen; VWR International). Temperature and apparent attenuation were determined with a portable density meter (DMA 35; Anton Paar, Graz, Austria).

\section{Culture-Dependent Microbiological Analyses}

\section{Selective Plating and Culturing}

The chilled samples were serially diluted in saline $(0.85 \%, \mathrm{~m} / \mathrm{v}$, $\mathrm{NaCl}$ ) and $100 \mu \mathrm{l}$ of each dilution was plated onto three selective agar media, which were chosen based on previous research (Spitaels et al., 2014, 2015): (i) modified de Man-RogosaSharpe (mMRS) agar medium for the enumeration and isolation of presumptive LAB after anaerobic incubation (AnaeroGen; Thermo Fisher Scientific, Waltham, MA, United States) at $20^{\circ} \mathrm{C}$ for 10 days to inhibit the growth of AAB; mMRS agar medium consisted of MRS agar (Oxoid, Basingstoke, Hampshire, United Kingdom) supplemented with a $0.2 \mathrm{ppm}$ vitamin solution (cobalamin, folic acid, nicotinic acid, pantothenic acid, pyridoxal phosphate, and thiamine) to enhance the chance to isolate nutritionally depending LAB species, and with $5 \mathrm{ppm}$ of amphotericin B (Sigma-Aldrich, Bornem, Belgium) and 200 ppm of cycloheximide (Sigma-Aldrich) to inhibit fungal growth; (ii) yeast extract-peptone-glucose (YPG) agar medium [2.0\% glucose (Merck, Darmstadt, Germany), 0.5\% yeast extract (Merck), $1.0 \%$ peptone (Oxoid), and 1.5\% agar (Oxoid); $\mathrm{m} / \mathrm{v}$ ] for the enumeration and isolation of presumptive yeasts after aerobic incubation at $30^{\circ} \mathrm{C}$ for 7 days; YPG medium was supplemented with 200 ppm of chloramphenicol to inhibit bacterial growth; and (iii) YPGc agar medium, i.e., YPG agar medium supplemented with $50 \mathrm{ppm}$ of cycloheximide to select for presumptive cycloheximide-resistant yeasts after aerobic incubation at $30^{\circ} \mathrm{C}$ for 7 days.

\section{Enumeration and Isolation of Colonies}

Agar media containing 30 to 300 colony forming units (CFU) were counted to determine the microbial community dynamics. In the case of $\mathrm{AAB}$, the average counts obtained on modified deoxycholate-mannitol-sorbitol (mDMS) agar medium for samples withdrawn from the same fermenting wort and maturing beer in the casks of the same lambic beer production processes but reported before were taken (De Roos et al., 2018). Subsequently, $10 \%$ of the total number of colonies from appropriate dilutions of the respective agar media were randomly picked to determine the culture-dependent microbial species diversity.

\section{Dereplication and Identification of Microbial Isolates by MALDI-TOF MS}

For dereplication and identification of the presumptive yeast and LAB isolates, each colony was sub-cultivated twice on its respective agar medium. The resulting third-generation colonies were used for matrix-assisted laser desorption ionization-time of flight mass spectrometry (MALDI-TOF MS) fingerprinting and mass spectra processing, as described previously for AAB (De Roos et al., 2018). Representative strains from each cluster, obtained through numerical analysis of the mass spectral profiles, were finally identified through comparative sequence analysis of the 16S rRNA gene for bacterial isolates and the internal transcribed spacer (ITS) region for yeast isolates, as described previously, and were considered identified to species level when the identity was higher than 97\% (Spitaels et al., 2014). The identities of the closest known relatives (type strains) were obtained using the basic local alignment search tool (BLAST) algorithm and by comparison with the GenBank database of the National Center for Biotechnology Information (NCBI, Bethesda, MD, United States ${ }^{1}$; Altschul et al., 1990). In the case AAB identities were needed, they were taken from the data on the AAB published previously (De Roos et al., 2018).

\section{Culture-Independent Microbiological Analyses}

Culture-independent analysis was performed on fermenting wort and maturing beer samples from cask 1 to check if some microbial groups were missed throughout sampling of the lambic beer

${ }^{1}$ http://blast.ncbi.nlm.nih.gov/ 
production processes, due to culture-dependent biases such as the appearance of microorganisms in a viable but not culturable state and/or the possibility that the isolation agar media used favored the cultivation of specific microorganisms.

\section{Total DNA Extraction}

To enable culture-independent microbial species diversity analysis, total DNA extracts were prepared from the cell pellets obtained from the original samples, following a method combining enzymatic, chemical, and mechanical treatments for cell lysis and phenol/chloroform/isoamyl alcohol treatment and column purification to extract and purify the DNA, as described before (Vermote et al., 2018).

\section{Amplification of Group-Specific Loci}

Amplification of group-specific loci was performed as described previously (De Bruyn et al., 2017). Therefore, specific loci of bacterial and fungal marker genes were amplified with primers purchased from Integrated DNA Technologies (Leuven, Belgium) through PCR. The V4 hypervariable region of the bacterial 16S rRNA gene was amplified with the primers F515 (5' -TCGTCGGCAGCGTCAGATGTGTATAAGAGACAGGTGT GCCAGCMGCCGCGGTAA-3') and R806 (5'-GTCTCGTGG GCTCGGAGATGTGTATAAGAGACAGG GACTACHVGGG TWTCTAAT-3') (Caporaso et al., 2011), both with a specific Illumina platform $5^{\prime}$ tag (underlined). The thermal PCR program was run under the following conditions: denaturation at $94^{\circ} \mathrm{C}$ for $3 \mathrm{~min}$, followed by 35 amplification cycles at $94^{\circ} \mathrm{C}$ for $45 \mathrm{~s}$ (denaturation), $50^{\circ} \mathrm{C}$ for $60 \mathrm{~s}$ (annealing), and $72^{\circ} \mathrm{C}$ for $90 \mathrm{~s}$ (extension), and final elongation at $72^{\circ} \mathrm{C}$ for $10 \mathrm{~min}$. The fungal ITS1 region of the rDNA was amplified with the primers BITS (5' -TCGTCGGCAGCGTCAGATGTGTATAAGAGACAGACCT GCGGARGGATCA- $\left.3^{\prime}\right)$ and B58S3 (5'-GTCTCGTGGGCTC GGAGATGTGTATAAGAGACAGGAGATCCRTTGYTRAAAG TT-3') (Bokulich and Mills, 2013), both with a specific Illumina platform $5^{\prime}$ tag (underlined). The thermal PCR program was run under the following conditions: denaturation at $95^{\circ} \mathrm{C}$ for $2 \mathrm{~min}$, followed by 40 amplification cycles at $95^{\circ} \mathrm{C}$ for $30 \mathrm{~s}$ (denaturation), $50^{\circ} \mathrm{C}$ for $30 \mathrm{~s}$ (annealing), and $72^{\circ} \mathrm{C}$ for $60 \mathrm{~s}$ (extension), and final extension at $72^{\circ} \mathrm{C}$ for $5 \mathrm{~min}$. A commercially available bacterial mock community (HM-782D; BEI Resources, Manassas, VA, United States) was amplified simultaneously with all samples to detect sequencing errors afterward. PCR sample mixtures were prepared and checked for the appropriate length, as described before (De Bruyn et al., 2017).

\section{High-Throughput Sequencing of the V4 and ITS1 Amplicons}

High-throughput sequencing of the V4 and ITS1 amplicons was performed, as described previously (De Bruyn et al., 2017). Therefore, the PCR amplicons obtained were purified using the Wizard SV gel and PCR clean-up system (Promega, Madison, WI, United States), following the manufacturer's instructions, after which they were eluted into $30 \mu$ l of nuclease-free water. Subsequently, they were subjected to size selection, using Agencourt AMPure XP PCR purification magnetic beads
(Beckman Coulter, Brea, CA, United States), according to the manufacturer's instructions, except that the relative amount of bead solution was changed to $1.1 \times$ for the BITS/B58S3 primer set, Eppendorf tubes were left to dry to the open air for maximally $3 \mathrm{~min}$, and elution was done into $30 \mu \mathrm{l}$ of nuclease-free water. The quality and concentrations of the amplicons were validated, as described before (De Bruyn et al., 2017). Finally, the V4 and ITS1 amplicons originating from the same sample were pooled in a 2:1 molar ratio and barcoded with the same index prior to sequencing. All samples were paired-end sequenced together with the amplified bacterial mock community, using the Illumina MiSeq platform (Illumina, San Diego, CA, United States) at the interuniversity VUB-ULB sequencing facility (BRIGHTcore, Jette, Belgium). Two Fastq files were obtained for each sample, containing all forward and reverse reads from both bacterial and fungal amplicons.

\section{Bioinformatics Analysis}

The two Fastq files of each sample (forward and reverse sequences), containing both bacterial V4 and fungal ITS1 sequences, were split into an additional two files, containing only forward and reverse sequences of the V4 and ITS1 regions, respectively, as described previously (De Bruyn et al., 2017). Therefore, different workflows were followed to process the bacterial and fungal sequences. For the bacterial V4 sequences (291 base pairs) of all samples, including the mock community, primers were removed (reverse primer for the forward sequences and forward primer for the reverse sequences, respectively) using the Cutadapt software (Martin, 2011), before subjection to the mothur software version 1.36.1 (Schloss et al., 2009). After generation of the contigs, the unique sequences were clustered into groups based on a maximum of two mismatches. Chimeric sequences were removed with the UCHIME algorithm (Edgar et al., 2011). The most abundant sequence of each group was chosen as the representative one and was taxonomically assigned by alignment against the bacterial 16S rRNA SILVA database (release 132) to remove non-bacterial reads. Afterward, the representative unique sequences that were assigned the same "order" taxon were clustered together. Within each cluster, multiple alignment analysis was performed to group the sequences into operational taxonomic units (OTUs) at a level of $97 \%$ identity. The sequence error rate was estimated in mothur by comparing the sequenced mock community against the in silico mock community. Regarding the fungal ITS1 sequences, which could vary in length, the forward and reverse sequences were trimmed using the Cutadapt software to avoid possible adapter read-throughs of ITS1 sequences shorter than the 300-base pair chemistry used by the Illumina platform (Gweon et al., 2015). The trimmed files were then quality-screened and further processed using the mothur software to generate contigs, as described before (De Bruyn et al., 2017). In particular, the unique sequences were classified taxonomically through comparison with the fungal UNITE_ITS1 database (v6_sh_99), and merged into OTUs when the taxonomic allocation was identical (Findley et al., 2013). 


\section{Substrate and Metabolite Target Analyses}

Substrate and metabolite concentrations were determined in the cell-free supernatants obtained from the original samples. Quantification was performed through external calibration, unless stated otherwise. All samples were both prepared and analyzed in triplicate.

\section{Determination of Simple Carbohydrate Concentrations}

The concentrations of fructose, glucose, maltose, and sucrose were measured by high-performance anion exchange chromatography coupled to pulsed amperometric detection (HPAEC-PAD) with internal standardization, as described before (De Roos et al., 2018). Briefly, an ICS3000 chromatograph (Dionex, Sunnyvale, CA, United States) equipped with a Carbopac $^{\mathrm{TM}}$ PA10 column (Dionex) and coupled to a pulsed amperometric detector (Dionex) was used. The same mobile phase and eluent gradient were applied. All samples were deproteinized, vortexed, centrifuged $\left(21,912 \times \mathrm{g}, 15 \mathrm{~min}, 4^{\circ} \mathrm{C}\right)$, and filtered $(0.2-\mu \mathrm{m}$ pore-size Whatman filters; GE Healthcare Life Sciences, Bucks, United Kingdom) before injection (10 $\mu \mathrm{l})$ into the column.

\section{Determination of Maltooligosaccharide Concentrations}

The concentrations of maltotriose, maltotetraose, maltopentaose, maltohexaose, and maltoheptaose were measured by HPAECPAD with internal standardization, as described before for maltotriose (De Roos et al., 2018). Briefly, an ICS3000 chromatograph (Dionex) equipped with a Carbopac ${ }^{\mathrm{TM}}$ PA100 column (Dionex) and coupled to a pulsed amperometric detector (Dionex) was used. The same mobile phase and eluent gradient were applied. All samples and standards were deproteinized, vortexed, centrifuged, and filtered, as described above, before injection $(10 \mu \mathrm{l})$ into the column.

\section{Determination of Ethanol and Short Chain Fatty Acid Concentrations}

The concentrations of ethanol, acetic acid, propionic acid, and butyric acid were measured by high-performance liquid chromatography with refractive index detection (HPLC-RI), applying external calibration, as described before for ethanol and acetic acid (De Roos et al., 2018). Briefly, a Waters chromatograph (Waters, Milford, MA, United States) equipped with an ICSep ICE ORH-801 column (Transgenomic North America, Omaha, NE, United States) and coupled to a RI detector (Waters) was used. The same mobile phase and eluent flow rate were used. All samples and standards were deproteinized, vortexed, centrifuged, and filtered, as described above, before injection $(30 \mu \mathrm{l})$ into the column.

\section{Determination of Lactic Acid Stereoisomer Concentrations}

The concentrations of $\mathrm{D}$ - and L-lactic acid were measured by HPLC with ultraviolet detection (HPLC-UV) and external calibration, based on a method described before, except that sample and standard preparations were deproteinized by adding $300 \mu \mathrm{l}$ of Carrez A solution [36 g/l of $\mathrm{K}_{4} \mathrm{Fe}(\mathrm{CN})_{6} \cdot 3 \mathrm{H}_{2} \mathrm{O}$ ] and $300 \mu \mathrm{l}$ of Carrez B solution $\left(72 \mathrm{~g} / \mathrm{l}\right.$ of $\left.\mathrm{ZnSO}_{4} .7 \mathrm{H}_{2} \mathrm{O}\right)$ to $600 \mu \mathrm{l}$ of analyte (Laureys and De Vuyst, 2014). They were analyzed with a Waters chromatograph equipped with a Shodex ORpak CRX-853 column (Showa Denko, Tokyo, Japan) and coupled to a UV detector operating at $253 \mathrm{~nm}$ (Waters). The mobile phase consisted of $10 \%$ acetonitrile (Fisher Chemical, Loughborough, United Kingdom) and $90 \% 1 \mathrm{mM} \mathrm{CuSO}_{4}$ (Merck) at a flow rate of $1 \mathrm{ml} / \mathrm{min}$. All samples and standards were vortexed, centrifuged, and filtered, as described above, before injection $(30 \mu \mathrm{l})$ into the column.

\section{Determination of Volatile Concentrations}

The concentrations of acetoin, 2,3-butanediol, 2,3-butanedione, ethyl acetate, ethyl lactate, isoamyl acetate, (iso)butyric acid, (iso)valeric acid, methyl-1-butanol, and propionic acid were measured by gas chromatography with flame ionization detection (GC-FID), applying internal standardization, as described before for ethyl acetate and acetoin (De Roos et al., 2018). Briefly, a Focus gas chromatograph (Interscience, Breda, Netherlands) equipped with a Stabilwax-DA column (Restek, Bellefonte, PA, United States) coupled to a FID-80 detector (Interscience) was used. All operating conditions were the same. All samples and standards were deproteinized, vortexed, centrifuged, and filtered as described above, before injection ( $1 \mu \mathrm{l}$; split 40$)$ into the column.

\section{Determination of Organic Acid Concentrations}

The concentrations of citric acid, gluconic acid, malic acid, and succinic acid were measured by ultra-performance liquid chromatography with tandem mass spectrometric detection (UPLC-MS/MS), applying external calibration, as described before for gluconic acid (De Roos et al., 2018). Briefly, an Acquity system chromatograph (Waters) equipped with a HSS T3 column (Waters) and coupled to a TQ tandem mass spectrometer with a ZSpray ${ }^{\mathrm{TM}}$ electrospray ionization source in negative ionization mode (Waters) was used. The same mobile phase and eluent gradient were applied. All samples and standards were deproteinized, vortexed, centrifuged, and filtered, as described above, before injection $(10 \mu \mathrm{l})$ into the column.

\section{Determination of Biogenic Amine Concentrations}

The concentrations of agmatine, cadaverine, histamine, 2phenylethylamine, putrescine, tryptamine, and tyramine were measured by UPLC-MS/MS, using multiple reaction monitoring and internal standardization. Preparation of samples and standards involved predilution in ultrapure water followed by deproteinization. For the latter, $600 \mu \mathrm{l}$ of acetonitrile (Fisher Chemical) was added to $300 \mu \mathrm{l}$ of analyte plus $300 \mu \mathrm{l}$ of internal standard (N,N-dimethylcyclohexylamine; Fluka Chemie, Buchs, Switzerland). They were analyzed with an Acquity chromatograph equipped with a HSS T3 column coupled to a TQ tandem mass spectrometer with a ZSpray ${ }^{\mathrm{TM}}$ electrospray ionization source used in positive ionization mode (Waters). The mobile phase consisted of $950 \mathrm{ml}$ of ultrapure water, $50 \mathrm{ml}$ of acetonitrile (Fisher Chemical), $10 \mathrm{mM}$ ammonium acetate 
(Merck), and $2 \mathrm{ml}$ of formic acid (Merck) (eluent A), and $50 \mathrm{ml}$ of ultrapure water, $950 \mathrm{ml}$ of acetonitrile, $10 \mathrm{mM}$ ammonium acetate, and $2 \mathrm{ml}$ of formic acid (eluent $\mathrm{B}$ ), at a flow rate of $0.3 \mathrm{ml} / \mathrm{min}$, with the following gradient: $0.0-1.0 \mathrm{~min}$, isocratic $100 \% \mathrm{~A} ; 1.0-1.6 \mathrm{~min}$, linear from 100 to $90 \% \mathrm{~A}$ and from 0 to $10 \% \mathrm{~B}$; $1.6-3.4 \mathrm{~min}$, isocratic $90 \% \mathrm{~A}$ and $10 \% \mathrm{~B} ; 3.4-3.5 \mathrm{~min}$, linear from 90 to $40 \% \mathrm{~A}$ and from 10 to $60 \% \mathrm{~B} ; 3.5-6.0 \mathrm{~min}$, isocratic $40 \% \mathrm{~A}$ and $60 \% \mathrm{~B} ; 6.0-6.2 \mathrm{~min}$, linear from 40 to $100 \% \mathrm{~A}$; and $6.2-7.5 \mathrm{~min}$, isocratic $100 \% \mathrm{~A}$. The following mass spectrometric settings were used: capillary voltage, $3.50 \mathrm{kV}$; cone voltage, $10-23 \mathrm{~V}$, depending on the biogenic amine detected; source temperature, $120^{\circ} \mathrm{C}$; desolvation temperature, $350^{\circ} \mathrm{C}$; cone gas flow, $50 \mathrm{l} / \mathrm{h}$; desolvation gas flow, $550 \mathrm{l} / \mathrm{h}$; and collision energy, 11-15 eV, depending on the biogenic amine detected. All samples and standards were vortexed, centrifuged, and filtered, as described above, before injection $(10 \mu \mathrm{l})$ into the column.

\section{RESULTS}

\section{Course of pH, Temperature, and Apparent Attenuation}

Two lambic beer production processes, carried out in two identical oak casks (biological duplicates), were studied as a function of time. The $\mathrm{pH}$, temperature, and apparent attenuation of the wort before its transfer into the casks was $4.3,17.1^{\circ} \mathrm{C}$, and $12.6^{\circ} \mathrm{P}$, respectively. Afterward, the $\mathrm{pH}$, temperature, and apparent attenuation profiles of the fermenting wort and maturing beer in both casks were comparable (Figure 1). The $\mathrm{pH}$ decreased during the first 2 weeks of fermentation from 4.3 to 3.8 , corresponding with enterobacterial and $\mathrm{AAB}$ growth, after which a slight increase was noticeable up to $\mathrm{pH} 4.0$ until month 3 , suggesting organic acid consumption by yeast species, before it dropped again from month 3 onward to values below $\mathrm{pH} 3.5$ at month 9, corresponding with $\mathrm{AAB}$ and LAB acidification (Figures 2-4). The temperature profiles followed the ambient temperature in the cellar and actually reflected the successive seasons. The apparent attenuation of the fermenting wort decreased rapidly in both casks during the first 7 weeks of fermentation, corresponding with ethanol production by yeasts during the main fermentation phase. It remained stable from week 7 until month 6 , indicating very low carbohydrate usage, after which a further decrease was noticeable from month 6 onward, corresponding with the maturation phase.

\section{Microbial Community Dynamics and Species Diversity}

The starting wort was spontaneously inoculated overnight during the coolship step and by the brewery equipment used, as indicated by the colony counts on the corresponding mMRS, YPG, and YPGc agar media. They represented presumptive LAB [log 3.0 $\mathrm{CFU} / \mathrm{ml}$, including enterobacteria (see below)], yeasts (log 4.0 $\mathrm{CFU} / \mathrm{ml}$ ), and cycloheximide-resistant yeasts (log $3.5 \mathrm{CFU} / \mathrm{ml})$, respectively (Figure 2). AAB were not present, as measured on mDMS agar medium (De Roos et al., 2018).
Based on the counts on these media as a function of time, the lambic beer production processes in both casks studied consisted of four distinctive phases (Figures 2-4). The first phase displayed growth of enterobacteria (first week of the production process), followed by a main fermentation phase (after $24 \mathrm{~h}$ till 7 weeks), an acidification phase (week 7 till month 9), and a maturation phase (from month 6 onward). Throughout these phases, a total of 391 bacterial and 1734 yeast isolates were obtained from mMRS agar medium (presumptive LAB) and YPG/YPGc agar media (presumptive yeasts/ cycloheximide-resistant yeasts), respectively, corresponding with 71 plated samples derived from the two lambic beer production casks examined (Figures 3, 4). Only small differences occurred between casks 1 and 2 regarding the microbial community dynamics and species diversity. No differences were found regarding the microbial species diversity for the different sampling heights (top, middle, and bottom of the fermenting wort and maturing beer in the casks).

Klebsiella oxytoca and Klebsiella variicola were most prevalent during the short enterobacterial phase $(\log 5.0 \mathrm{CFU} / \mathrm{ml})$. These bacteria were isolated from mMRS agar media, indicating that mMRS was not specific enough to isolate LAB solely. During this first phase, a first peak of AAB was found $(\log 3.5 \mathrm{CFU} / \mathrm{ml})$, whereby Acetobacter orientalis was most prevalent (Figures 2-4). High counts of both cycloheximide-resistant yeasts (up to almost $\log 6.0 \mathrm{CFU} / \mathrm{ml}$ ), among which Hanseniaspora uvarum was most prevalent, and cycloheximide-sensitive yeasts (up to almost $\log 7.0 \mathrm{CFU} / \mathrm{ml}$ ), among which $S$. cerevisiae was most prevalent, were found during the initial stages of the main fermentation phase (Figures 2-4). During the first days of fermentation, both $H$. uvarum and $S$. cerevisiae were present in nearly equal counts (up to $\log 6.0 \mathrm{CFU} / \mathrm{ml}$ ). Upon further progress of the fermentation, the number of cycloheximideresistant yeasts decreased rapidly (to $\log 4.0 \mathrm{CFU} / \mathrm{ml}$ ), and their species diversity shifted from H. uvarum to Kazachstania species. The number of cycloheximide-sensitive yeasts reached a maximum after 3 days of fermentation and remained stable for 3 weeks $(\log 7.0 \mathrm{CFU} / \mathrm{ml})$. Culture-independently, through amplicon sequencing of samples taken from the fermenting wort at the middle of cask 1, mainly Saccharomyces and Cellulosimicrobium species were found during the first month of fermentation (Figure 5). The occurrence of Cellulosimicrobium overlapped with the enterobacterial phase and lasted 2 weeks longer. During the final weeks of the main fermentation phase, the most prevailing yeast species shifted from $S$. cerevisiae to Saccharomyces kudriavzevii.

When the number of yeasts decreased, an increase in AAB was found (up to $\log 6.5 \mathrm{CFU} / \mathrm{ml}$ ), among which Acetobacter pasteurianus was most prevalent (Figures 2-4), and this marked the start of the acidification phase. From month 6 (cask 1) and 7.5 (cask 2) onward, LAB could be isolated, giving counts up to almost log 6.0 CFU/ml (Figure 2). All LAB isolated from mMRS agar media were identified as $P$. damnosus, except at month 24 in cask 1 wherein Lactobacillus buchneri was found at low counts ( $\log 3.0 \mathrm{CFU} / \mathrm{ml}$; Figure 3). These culture-dependent data were in agreement with those obtained from the culture-independent analysis performed on samples taken from the fermenting wort at the middle of cask 1, for which $\mathrm{LAB}$ were retrieved from 
A

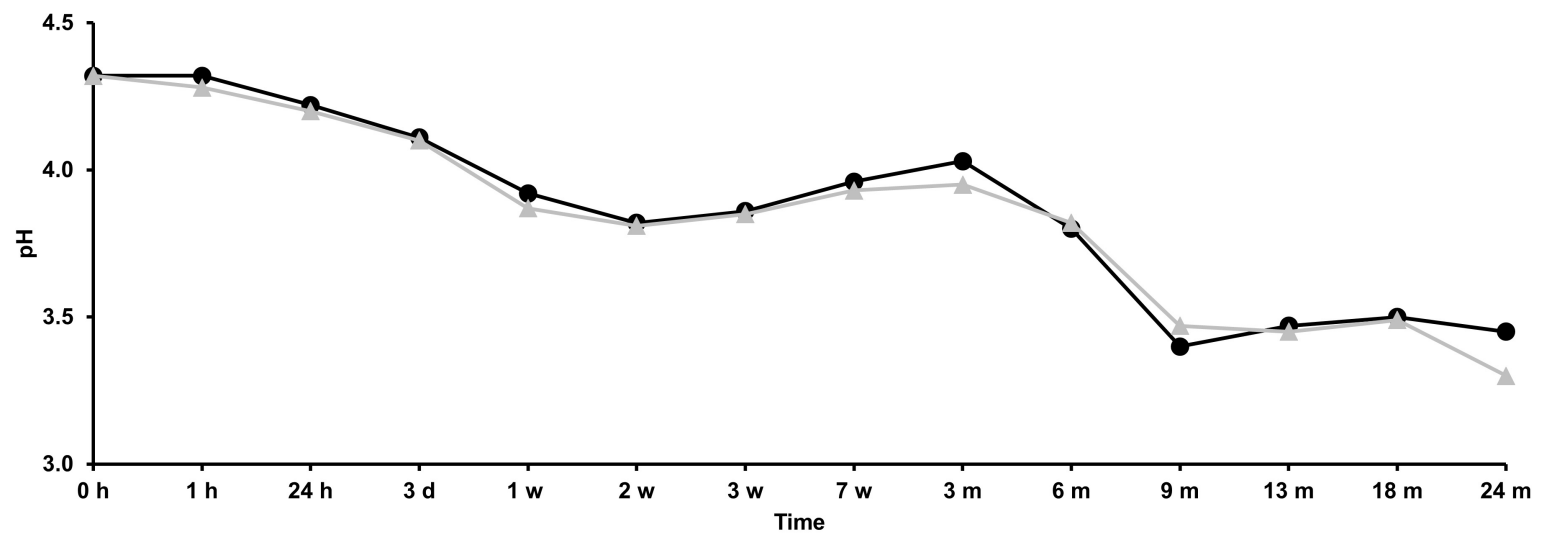

B

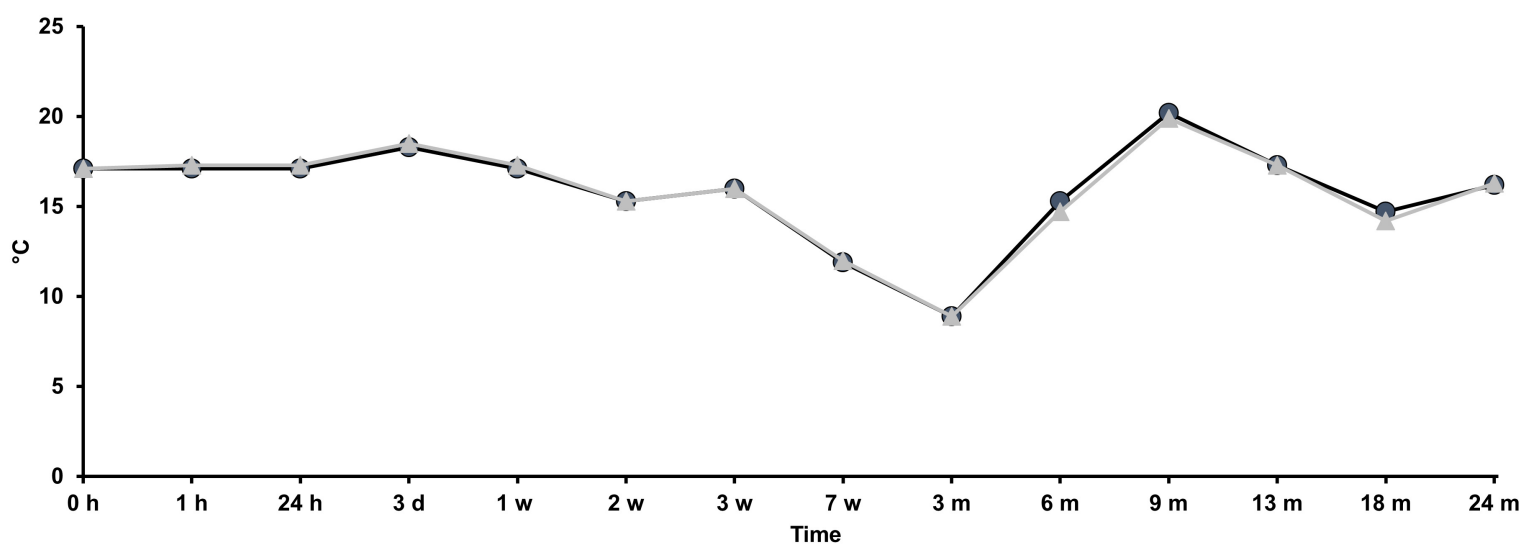

C

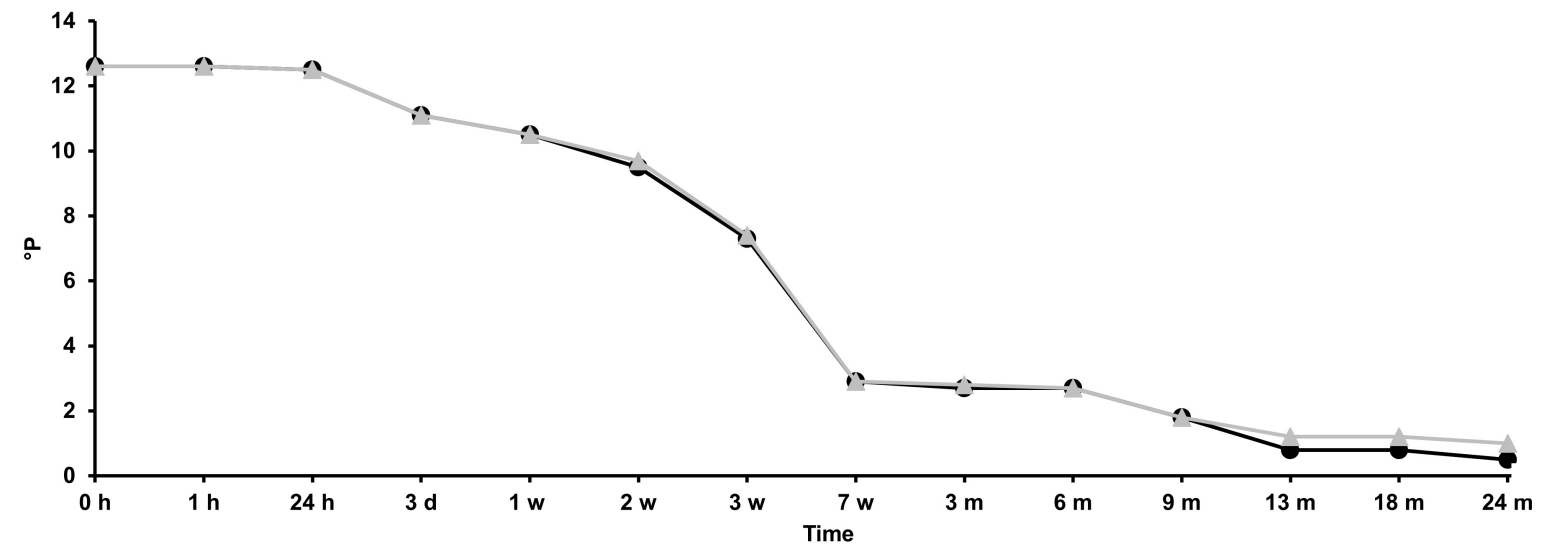

FIGURE 1 | Course of the physicochemical parameters $\mathrm{pH}$ (A), temperature (B), and apparent attenuation (C) in the fermenting wort and maturing beer during the 24-month lambic beer production processes carried out in two identical oak casks. Cask 1 (•) and cask $2(\mathbf{\Lambda})$.

month 6 onward (Figure 5). Together with the isolation of high numbers of $\mathrm{LAB}$, also yeast species started to increase again (up to $\log 6.0 \mathrm{CFU} / \mathrm{ml}$; Figure 2), and pellicle formation became visible.

From month 9 onward, the number of LAB decreased (below $\log 2.0 \mathrm{CFU} / \mathrm{ml}$ ) and oxidative yeasts such as Dekkera species and
Pichia membranifaciens co-occurred, as shown by the culturedependent analysis of casks 1 and 2 (Figures 3,4) and the cultureindependent analysis of cask 1 (Figure 5). Dekkera species could be isolated from both YPG and YPGc agar media from months 6 and 9 onward, in casks 1 and 2, respectively, giving counts of up to almost $\log 6.0 \mathrm{CFU} / \mathrm{ml}$ (Figures 2-4). This indicated the 

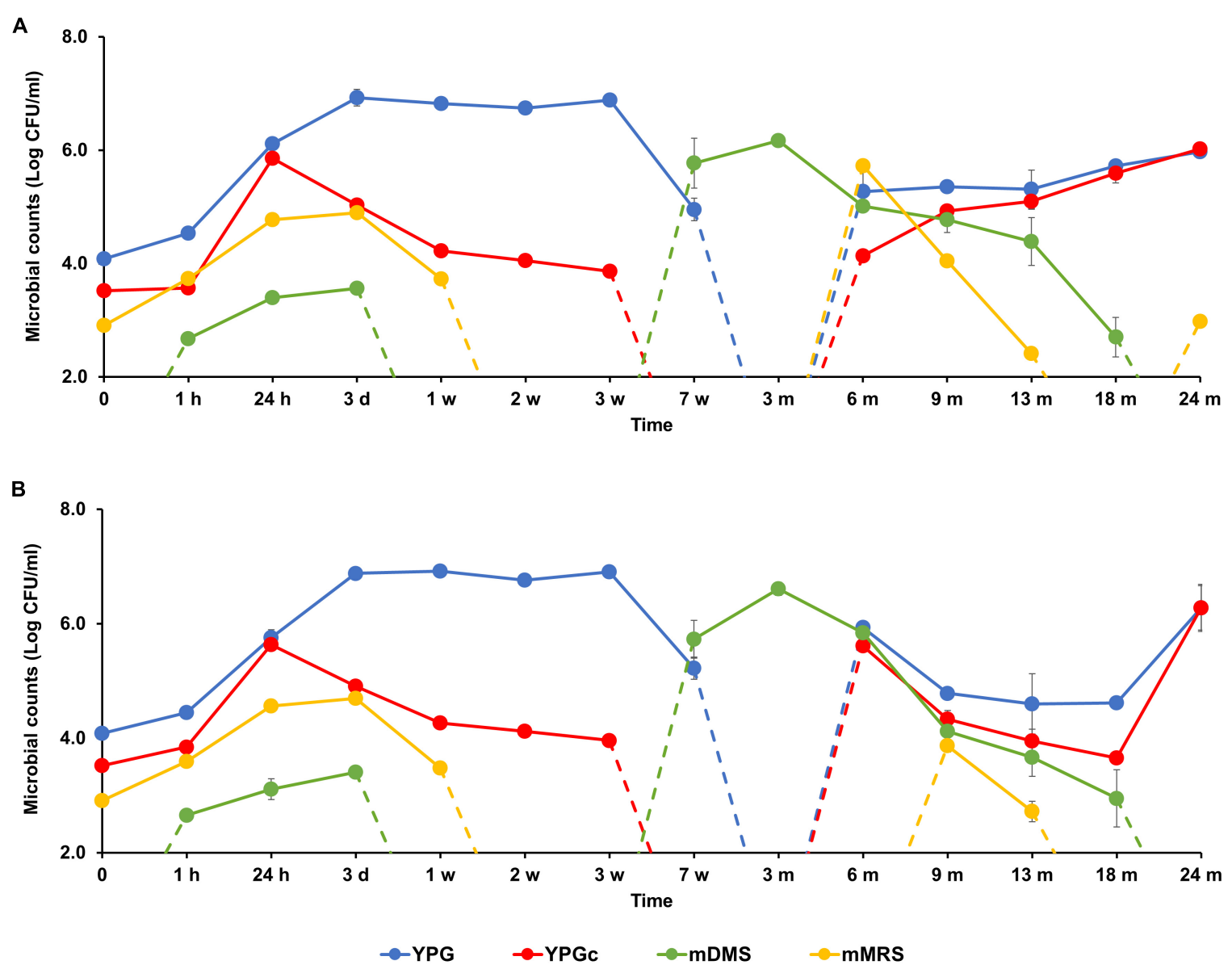

FIGURE 2 | Counts of presumptive yeasts, cycloheximide-resistant yeasts, acetic acid bacteria (AAB), and lactic acid bacteria (LAB), as plated on yeast-peptone-glucose (YPG) agar medium, yeast-peptone-glucose agar medium supplemented with cycloheximide (YPGc), modified deoxycholate-mannitol-sorbitol (mDMS) agar medium, and modified de Man-Rogosa-Sharpe (mMRS) agar medium, respectively, during the 24-month lambic beer production processes carried out in two identical oak casks. Since no differences were found regarding yeast and $L A B$ enumerations for the different sampling heights (top, middle, and bottom of the fermenting wort and maturing beer in the casks), average counts are reported. For the AAB, the average was taken of the counts from fermenting wort and maturing beer samples taken at the top, middle, and bottom of the same casks during the same lambic beer production processes reported before (De Roos et al., 2018). Cask 1 (A) and cask 2 (B).

start of the maturation phase. From month 18 onward, Dekkera species shifted from D. bruxellensis to Dekkera custersianus, possibly reflecting a higher stress tolerance from the latter yeast species toward the environmental conditions. In cask 1 , however, P. membranifaciens could be isolated from month 6 onward, although small numbers were probably present as early as month 3 , given its detection by culture-independent analysis of the maturing beer in cask 1 from that time point on. In cask 2, P. membranifaciens was only isolated from month 9 onward. Opportunistic yeast contaminants such as Ogataea species were occasionally detected as well.

\section{Substrate Consumption and Metabolite Production}

Concentrations and profiles of substrates and metabolites were comparable for the biological duplicates analyzed, unless stated otherwise. Since no differences were found regarding substrate and metabolite concentrations for the different sampling heights, concentrations measured in the fermenting wort and maturing beer at the top, middle, and bottom of the casks were averaged.

The initial wort was rich in carbohydrates, among which glucose $(8.0 \mathrm{~g} / \mathrm{l})$, fructose $(2.5 \mathrm{~g} / \mathrm{l})$, sucrose $(4.0 \mathrm{~g} / \mathrm{l})$, maltose $(60.0 \mathrm{~g} / \mathrm{l})$, maltotriose $(12.0 \mathrm{~g} / \mathrm{l})$, and higher maltooligosaccharides $(<4.0 \mathrm{~g} / \mathrm{l})$, were the most abundant (Figure 6). Organic acids, among which lactic acid (1.2 g/l), citric acid (250 mg/l), malic acid $(200 \mathrm{mg} / \mathrm{l})$, and gluconic acid (70 mg/l), were also present (Figure 7).

During the enterobacterial phase, no short-chain or branchedchain fatty acids were found, indicating limited growth of the background microbiota. During the main fermentation phase (first 7 weeks), mono-, di-, and trisaccharides were nearly completely depleted. The mono- and disaccharides were consumed sequentially (Figure 6). Sucrose was depleted after 3 days of fermentation, followed by the depletion of glucose and fructose after 1 and 2 weeks, respectively, which corresponded 


\section{A}

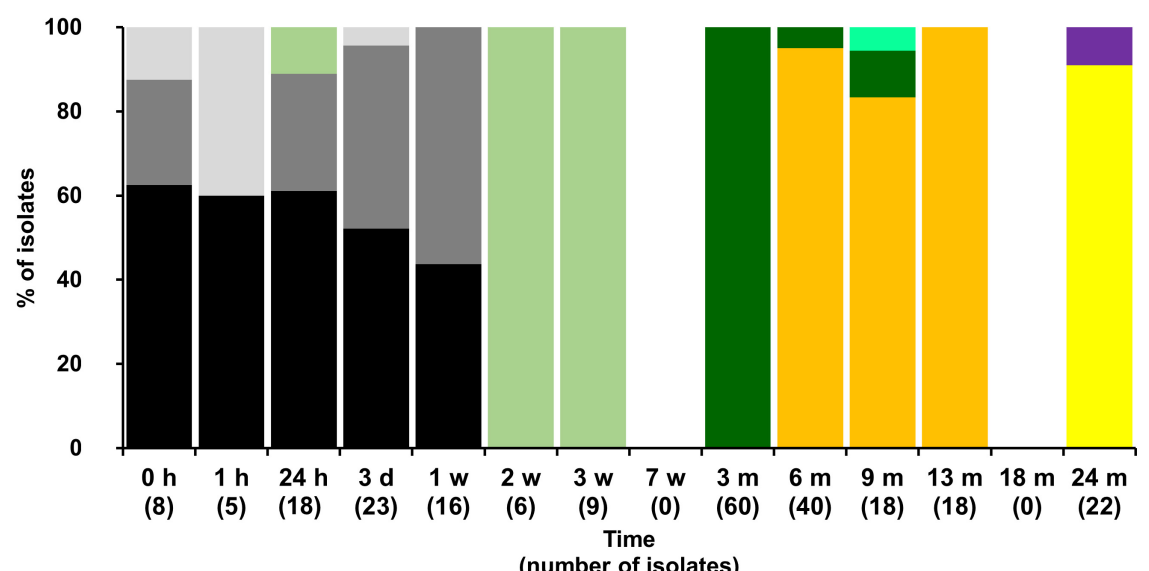

- Klebsiella variicola

- Klebsiella oxytoca

Enterobacter cloacae

Acetobacter orientalis

Pediococcus damnosus

- Acetobacter pasteurianus

Acetobacter lambici

Lactobacillus buchneri

- Unidentified strain

B

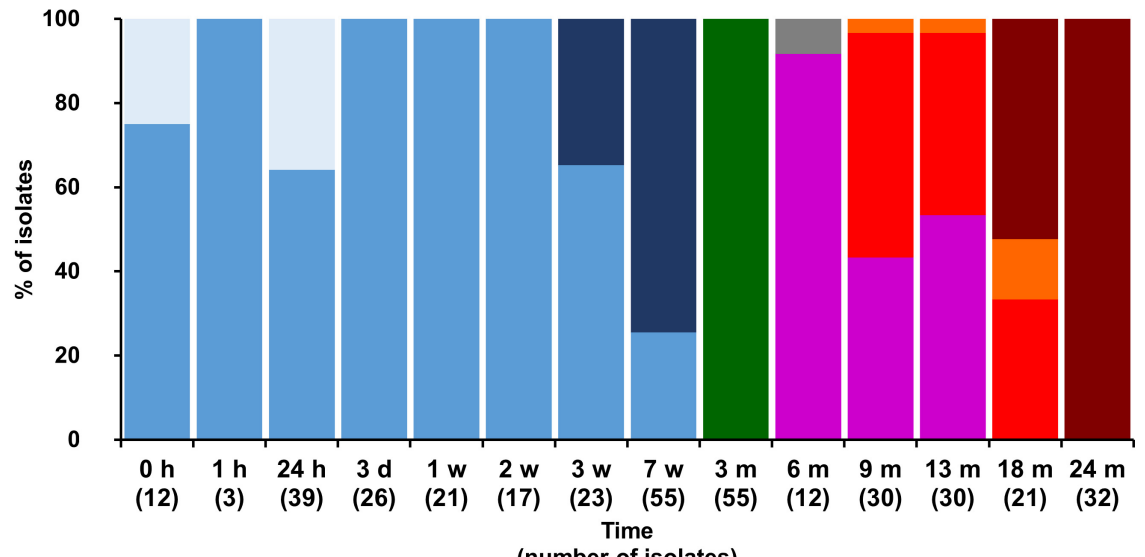

- Saccharomyces cerevisiae

Hanseniaspora uvarum

- Saccharomyces kudriavzevii

- Acetobacter pasteurianus

- Pichia membranifaciens

- Ogataea

- Dekkera bruxellensis

- Dekkera anomala

- Brettanomyces custersianus

C

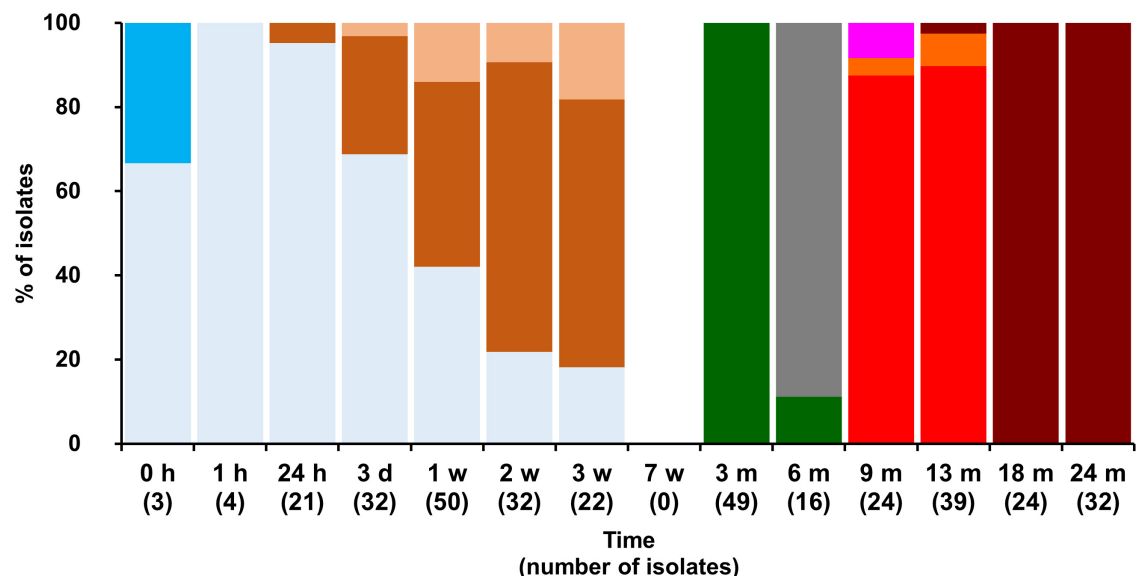

Hanseniaspora uvarum

- Yarowia lipolitica

- Kazachstania bulderi

Kazachstania unispora

- Acetobacter pasteurianus

- Ogataea

- Dekkera bruxellensis

- Dekkera anomala

- Candida

- Brettanomyces custersianus

FIGURE 3 | Community dynamics of the LAB (A), yeast (B), and cycloheximide-resistant yeast (C) species during the 24-month lambic beer production process carried out in cask 1. The following bacterial species were identified: Klebsiella variicola (GenBank accession number NR_025635.1), Klebsiella oxytoca (NR_112010.1), Acetobacter orientalis (NR_028625.1), Enterobacter cloacae (CP017184.1), Pediococcus damnosus (NR_042087.1), Acetobacter pasteurianus (CP015168.1), Acetobacter lambici (HG329531.1), and Lactobacillus buchneri (NR_041293.1). The following yeast species were identified: Saccharomyces cerevisiae (KC881067.1), Hanseniaspora uvarum (KY103558.1), Saccharomyces kudriavzevii (NR_111355.1), Pichia membranifaciens (NR_111195.1), Ogataea sp. (KY104429.1), Dekkera bruxellensis (KY103308.1), Dekkera anomala (KY103306.1), Dekkera custersianus (NR_138184.1). Yarrowia lipolytica (KY105973.1), Kazachstania bulderi (NR_138198.1), Kazachstania unispora (KY103682.1), and Candida sp. (NR_137645.1). 


\section{A}

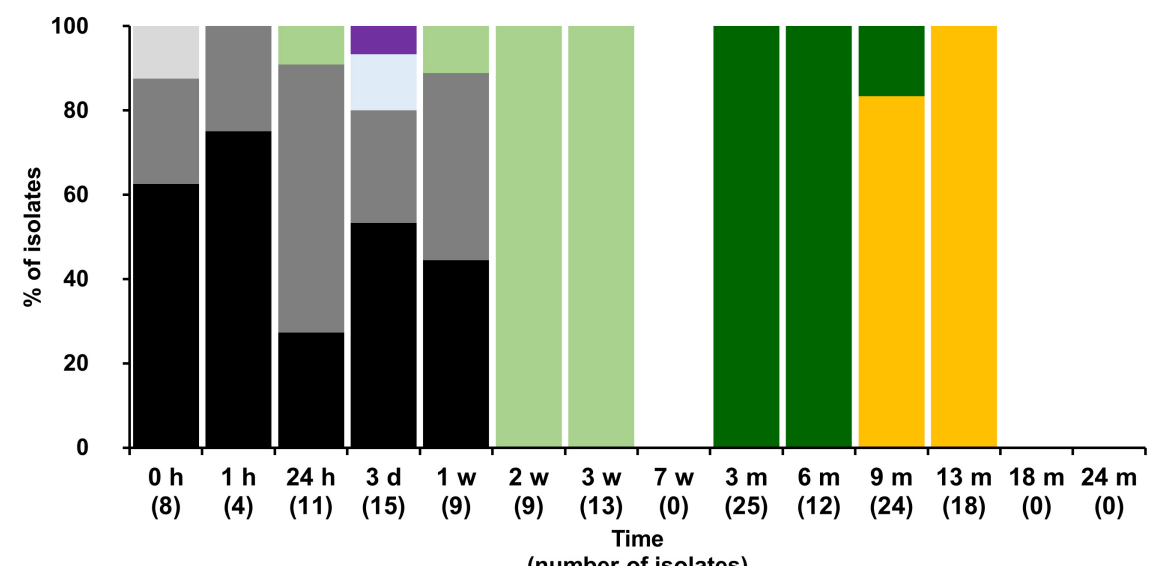

- Klebsiella variicola

- Klebsiella oxytoca

Enterobacter cloacae

Hanseniapora uvarum

Acetobacter orientalis

- Pediococcus damnosus

- Acetobacter pasteurianus

-Unidentified

(number of isolates)

B

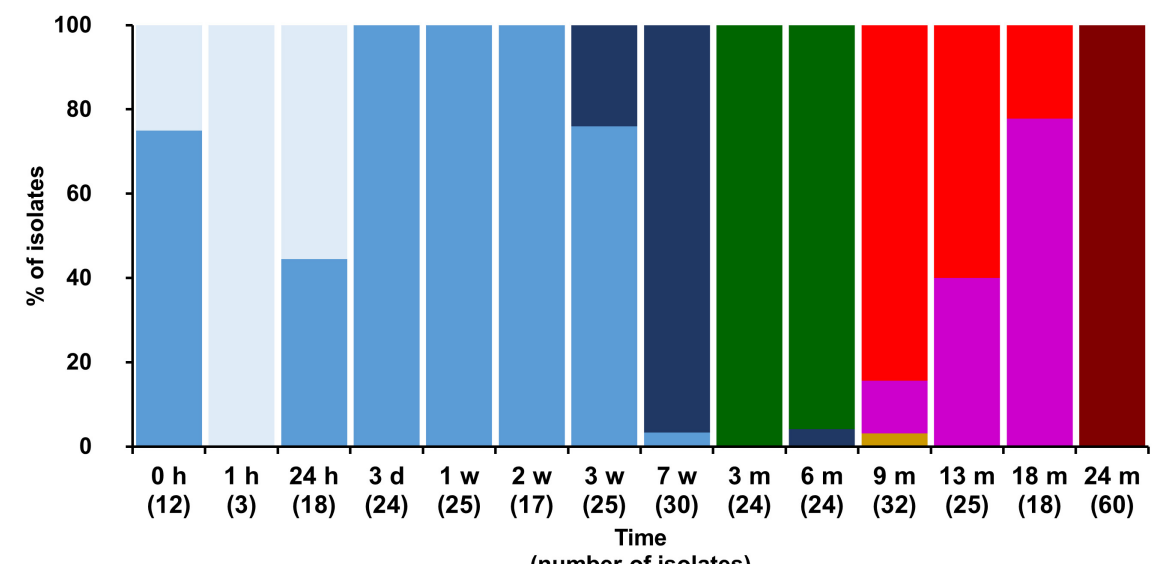

- Saccharomyces cerevisiae

\section{Hanseniaspora uvarum}

- Saccharomyces kudriavzevii

- Acetobacter pasteurianus

-Wickerhamomyces anomalus

- Pichia membranifaciens

- Dekkera bruxellensis

- Brettanomyces custersianus

C

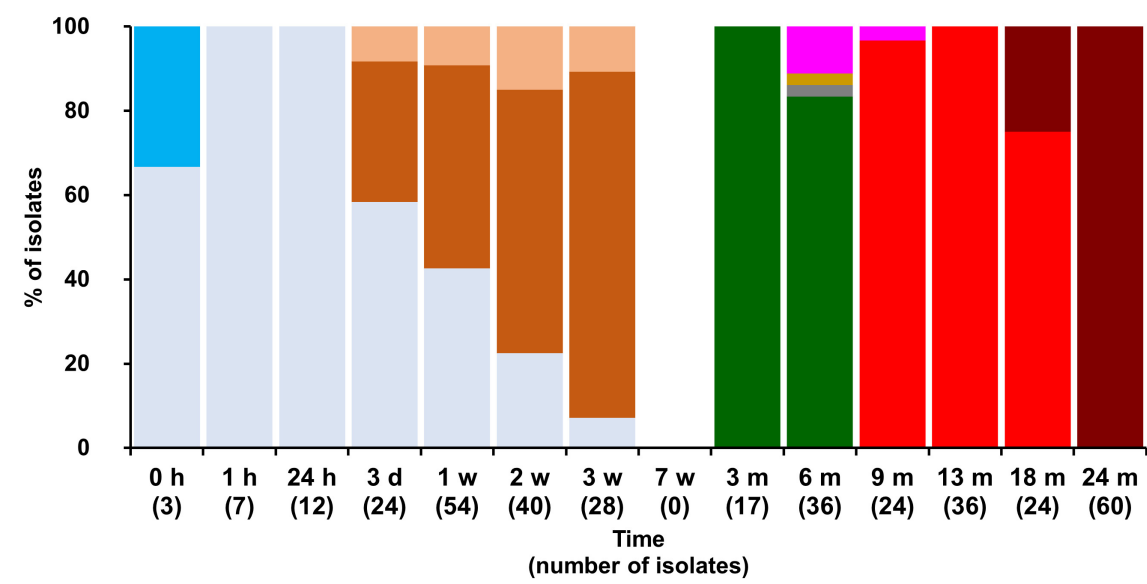

Hanseniaspora uvarum

- Yarowia lipolitica

- Kazachstania bulderi

Kazachstania unispora

- Acetobacter pasteurianus

- Ogataea

- Dekkera bruxellensis

- Debaryomyces hansenii

- Candida

- Brettanomyces custersianus

FIGURE 4 | Community dynamics of the LAB (A), yeast (B), and cycloheximide-resistant yeast (C) species during the 24-month lambic beer production process carried out in cask 2. The following microbial species were identified: Klebsiella variicola (GenBank accession number NR_025635.1), Klebsiella oxytoca (NR_112010.1), Enterobacter cloacae (CP017184.1), Hanseniaspora uvarum (KY103558.1), Acetobacter orientalis (NR_028625.1), Pediococcus damnosus (NR_042087.1), Acetobacter pasteurianus (CP015168.1), Saccharomyces cerevisiae (KC881067.1), Saccharomyces kudriavzevii (NR_111355.1),

Wickerhamomyces anomalus (KY105894.1), Pichia membranifaciens (NR_111195.1), Dekkera bruxellensis (KY103308.1), Dekkera custersianus (NR_138184.1), Yarrowia lipolytica (KY105973.1), Kazachstania bulderi (NR_138198.1), Kazachstania unispora (KY103682.1), Ogataea sp. (KY104429.1) Debaryomyces hansenii (KY103230.1), and Candida sp.(NR_137645.1). 


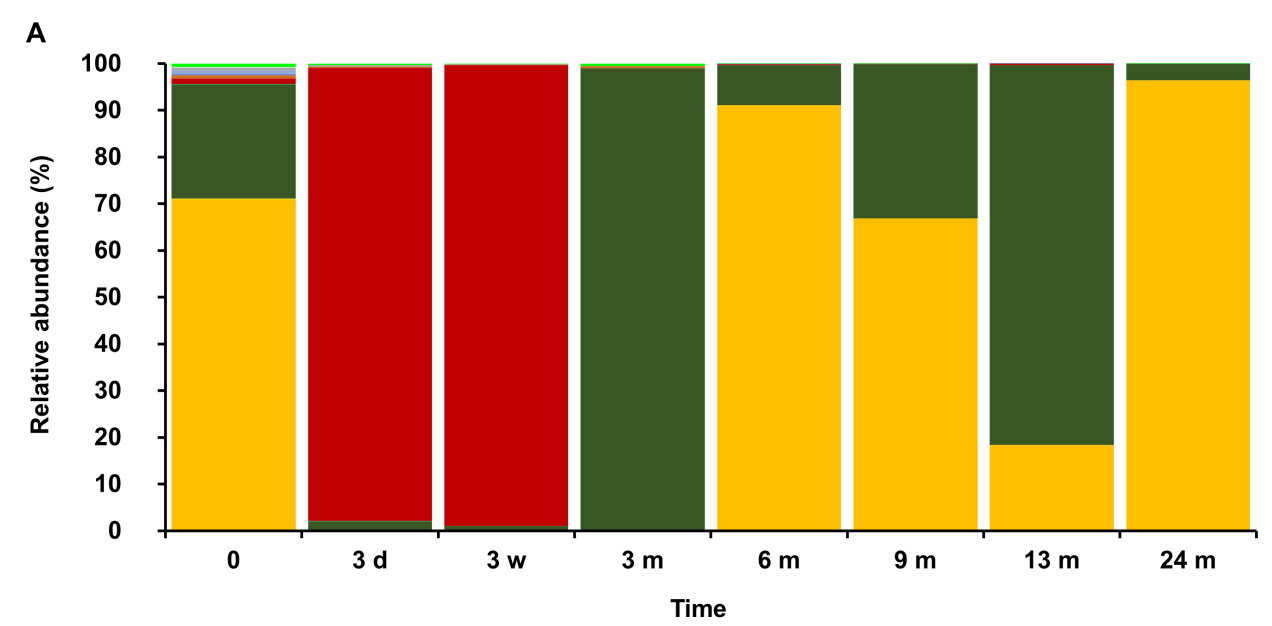

\section{Pediococcus \\ Lactobacillus \\ - Acetobacter \\ - Gluconobacter \\ - Cellulosimicrobium \\ Acinetobacter \\ - Staphylococcus \\ Sphingomonas \\ Pseudomonas \\ Corynebacterium \\ - Others}

B

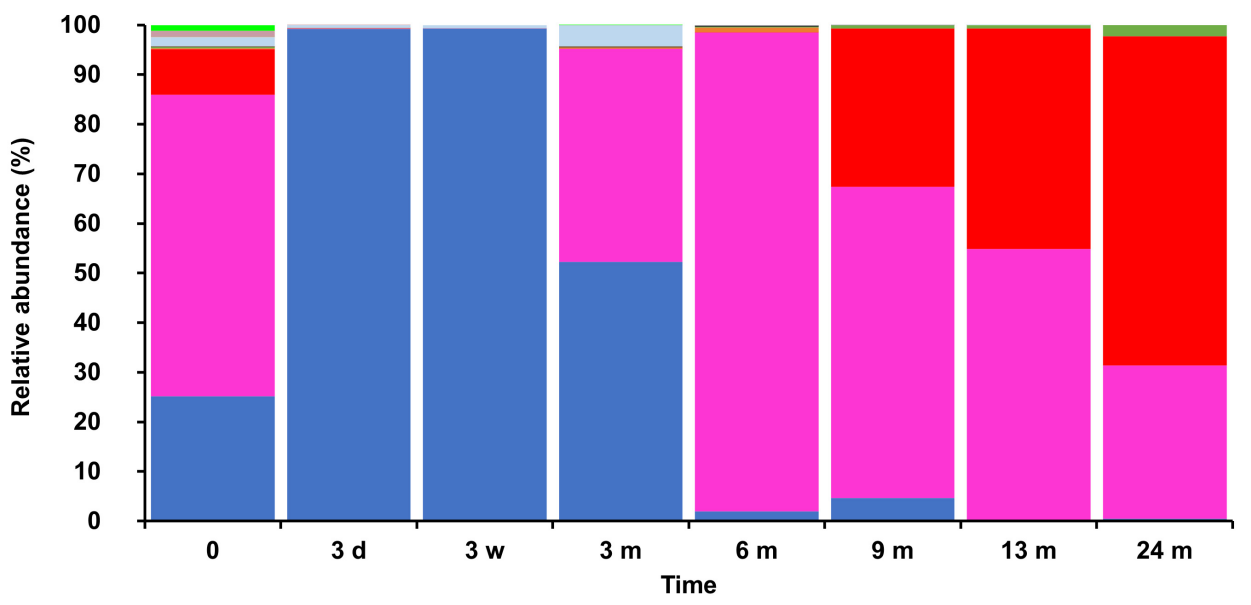

- Saccharomyces

- Pichia

- Dekkera

- Candida

Kregervanrija

- Penicillium

- Wickerhamomyces

Hanseniaspora

Meyerozyma

Trichosporon

Dipodascus

Others

FIGURE $\mathbf{5}$ | Relative abundances of bacterial (A) and fungal (B) operational taxonomic units obtained by amplicon sequencing of fermenting wort and maturing beer samples taken from the middle of the cask of the 24-month lambic beer production process conducted in cask 1.

with the enterobacterial and early main fermentation phases. Maltose showed a gradual degradation until it was completely depleted after week 7, coinciding with the alcoholic fermentation phase. Maltotriose followed the same degradation profile as maltose, indicating the ability of both maltose and maltotriose uptake and consumption by the strains of the Saccharomyces species present. The exhaustion of both maltose and maltotriose coincided with a decrease in Saccharomyces species counts. The higher maltooligosaccharides (from maltotetraose to maltoheptaose) were degraded simultaneously over time, starting from week 3 (Figure 6). Due to the continuous degradation of maltooligosaccharides, combined with the lack of consumption of mono-, di-, and trisaccharides, the concentrations of glucose, maltose, and maltotriose increased from week 7 until month 6 , corresponding with the acidification phase (Figure 6).

During the main fermentation phase (first 7 weeks), mostly yeast-associated metabolites such as ethanol, methyl-1-butanol, and succinic acid were produced (Figure 8). From week 3 onward, the most abundant esters of lambic beer, namely ethyl lactate and ethyl acetate, were produced until maximal concentrations of over $100 \mathrm{mg} / \mathrm{l}$ and around $300 \mathrm{mg} / \mathrm{l}$, respectively. Isoamyl acetate was not found. Acetoin was produced from 7 weeks until 6 months of fermentation, corresponding with $\mathrm{AAB}$ growth, reaching concentrations between 70 and $80 \mathrm{mg} / \mathrm{l}$. Together with the decline of $\mathrm{AAB}$ counts, acetoin concentrations decreased after 6 months, indicating consumption by the yeast species present during the maturation phase. 2,3-Butanediol and 2,3-butanedione were not found during the entire production process, indicating no further and full conversion of acetoin, respectively. Acetic acid was produced from week 7 onward, coinciding with the acidification and maturation phases, exceeding concentrations of $2.0 \mathrm{~g} / \mathrm{l}$ (Figure 7). From month 3 and 6 onward, lactic acid was produced in the fermenting wort of casks 1 and 2, respectively. Both Dand L-lactic acid were produced in nearly equal concentrations $(2.0 \mathrm{~g} / \mathrm{l})$. Coinciding with the production of lactic acid, malic acid was rapidly depleted after the occurrence of LAB at months 6 and 9 in casks 1 and 2, respectively. After being nearly completely depleted, the concentrations of malic acid slightly increased during the maturation phase, indicating limited malic 


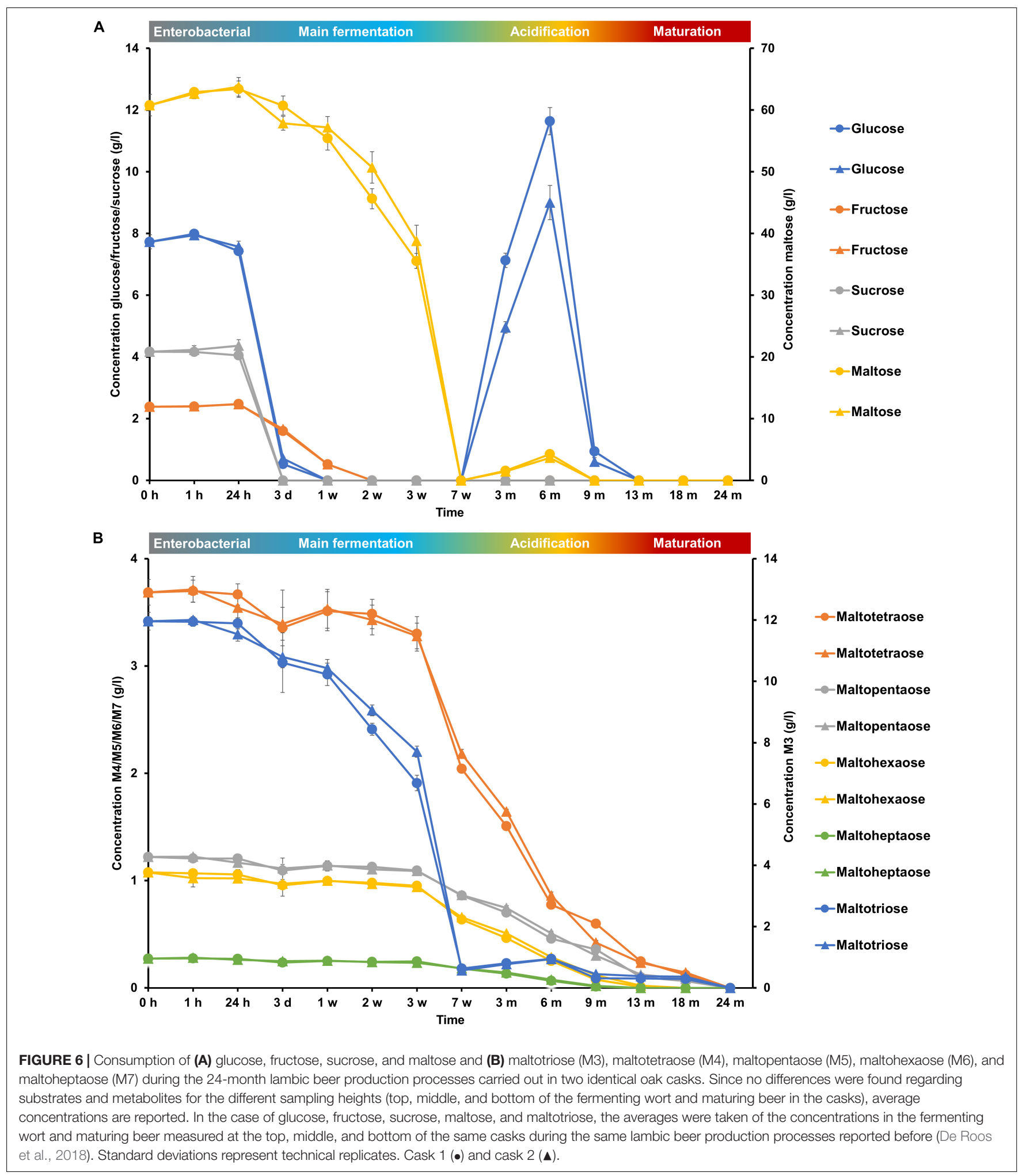

acid production by the yeast species present. In contrast, citric acid was not metabolized. Gluconic acid was present from the start of the fermentation and a slight increase was noticeable from week 7 until month 6 , coinciding with the presence of AAB.
The initial wort contained some biogenic amines at low concentrations, such as agmatine $(9 \mathrm{mg} / \mathrm{l})$, putrescine $(8 \mathrm{mg} / \mathrm{l})$, and cadaverine $(3 \mathrm{mg} / \mathrm{l})$ (Figure 9). Cadaverine mainly increased during the first week of fermentation, reaching concentrations 


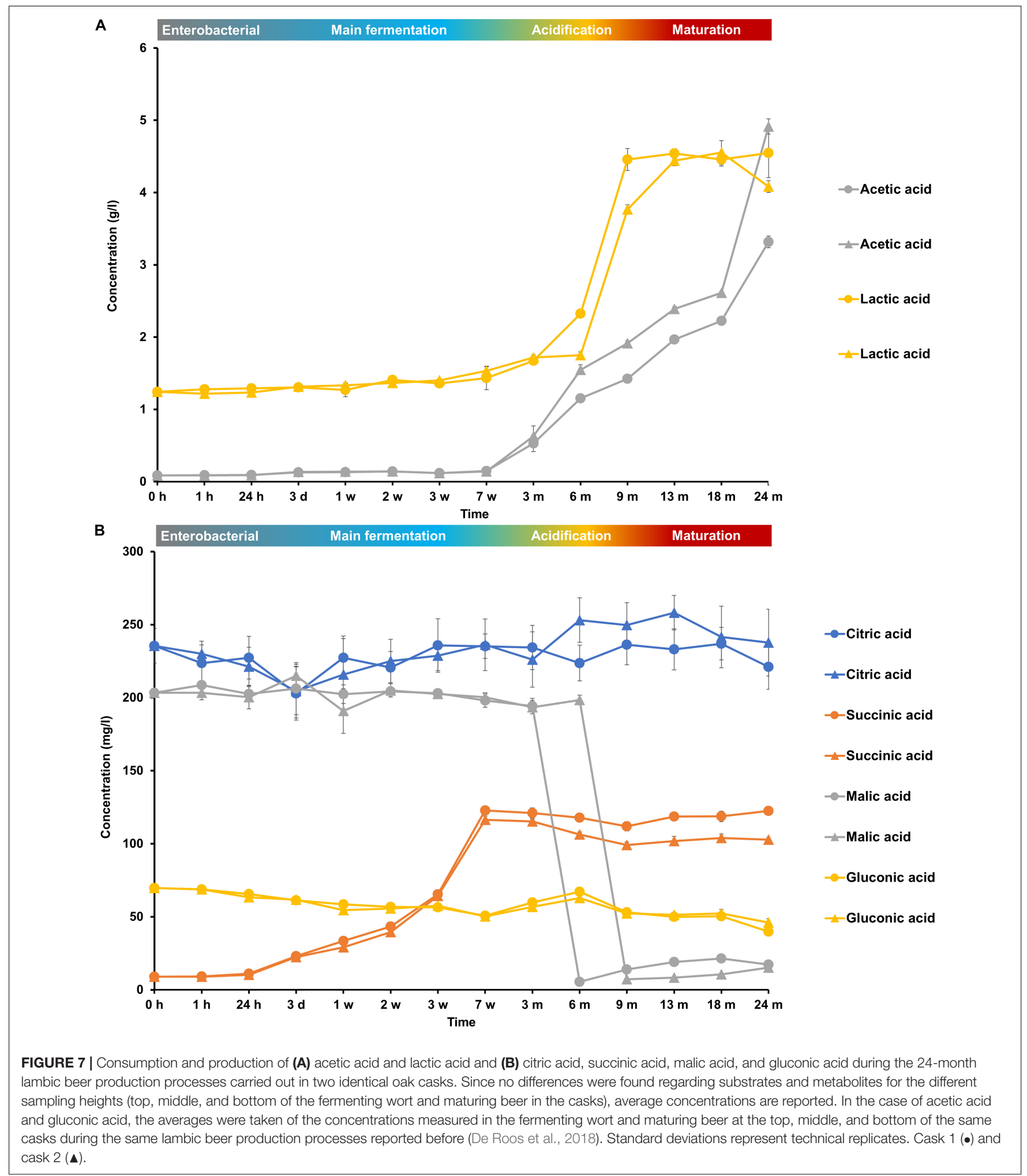

of over $30 \mathrm{mg} / \mathrm{l}$, indicating its production by the enterobacteria present. Agmatine was completely depleted after month 9 of fermentation in cask 2, whereas it was only partly consumed in cask 1 during the first days of fermentation mainly. Concentration changes of putrescine were negligible throughout the lambic beer production processes. Histamine was produced mainly during the acidification phase after months 3 and 6 in casks 1 and 2, respectively, indicating 


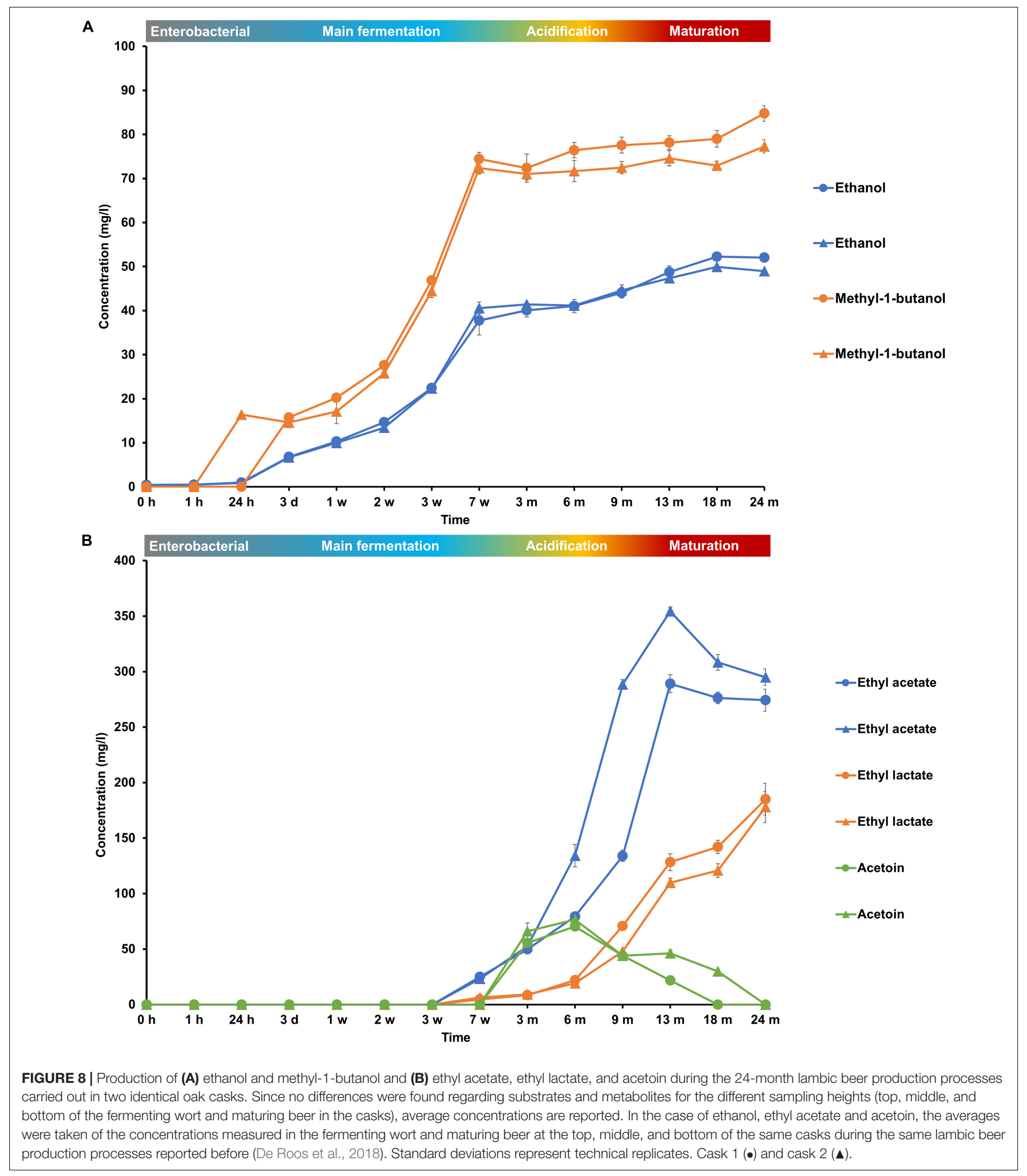

its production by the LAB species present. Histamine reached concentrations of 18 and $17 \mathrm{mg} / \mathrm{l}$ in casks 1 and 2, respectively. Tyramine was the most prevalent biogenic amine present in both casks at the end of the maturation phase and was mainly produced after months 6 and 18 in casks 1 and 2, respectively, reaching concentrations of $34 \mathrm{mg} / \mathrm{l}$ and $42 \mathrm{mg} / \mathrm{l}$, respectively. 2-Phenylethylamine and tryptamine were never found. 


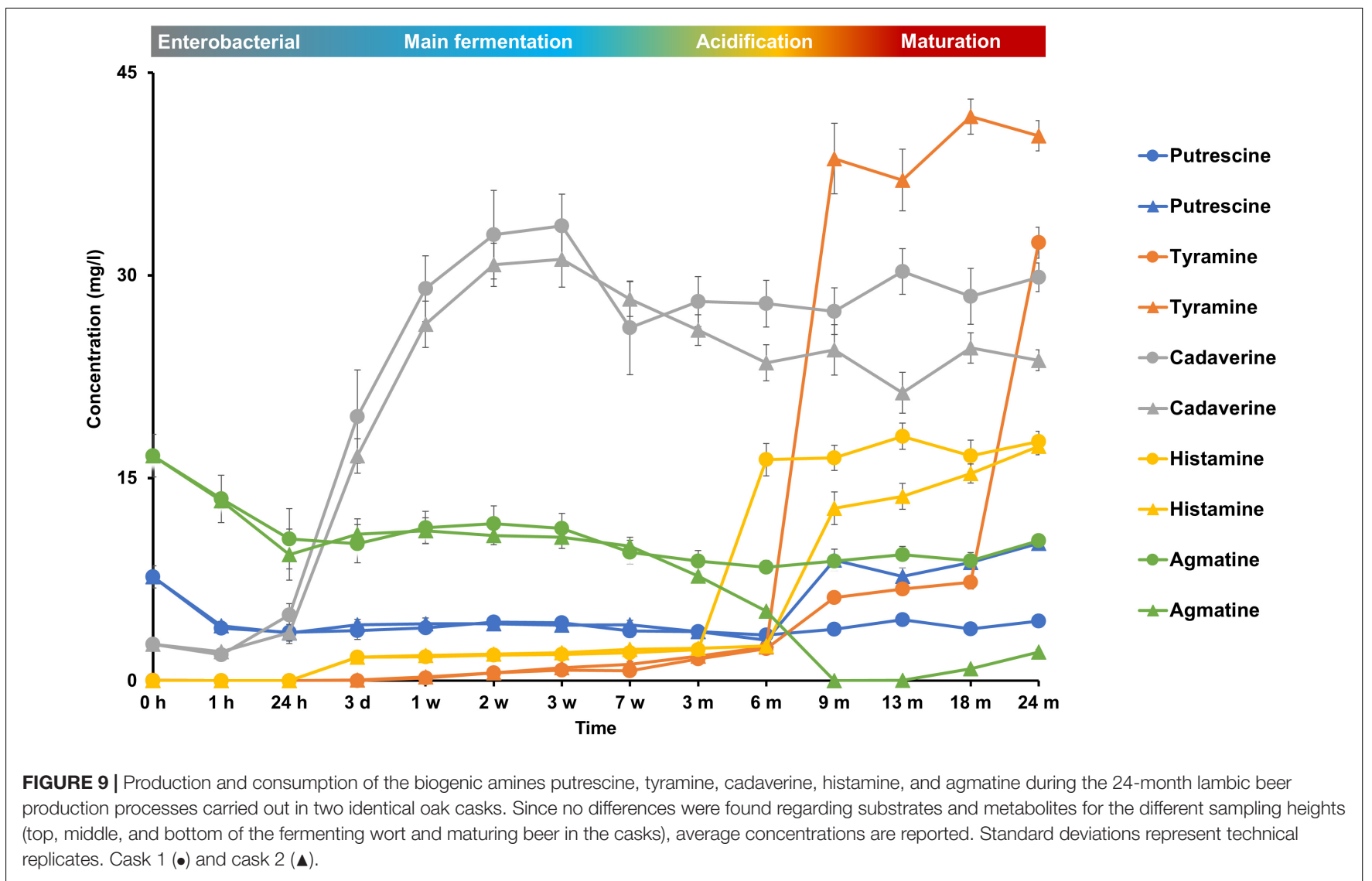

\section{DISCUSSION}

Traditional lambic beer production processes are characterized by a microbial succession of four groups of microorganisms (Verachtert and Iserentant, 1995; Verachtert and Derdelinckx, 2005; Spitaels et al., 2014; De Roos et al., 2018). Recently performed studies mainly dealt with the microbiology of lambic beer production processes performed in a traditional and common brewery, leading to new insights into this complex spontaneous fermentation and maturation process, but did not tackle physicochemical and metabolomic parameters (Spitaels et al., 2014, 2015). Only the impact of the growth of and substrate consumption and metabolite production by AAB has been underlined recently (De Roos et al., 2018). Therefore, the present study unraveled the dynamics, species diversity, and substrate consumption and metabolite production kinetics of all microbial groups involved in the four consecutive phases of a Belgian, traditional lambic beer production process carried out for 24 months. Moreover, although latest studies have shown that no enterobacterial phase occurs when the wort is acidified (Spitaels et al., 2015), the four distinctive phases of a lambic beer production process were retrieved during the production processes of the present study, albeit that the enterobacterial phase was shorter, spanning the first week of fermentation only, due to the application of manual wort acidification (with lactic acid) before the coolship step. Whether the extent of acidification (depending on the type of acidulant, concentration applied, etc.) or air inoculation (environmental temperature, wort cooling speed, etc.) was responsible for this short enterobacterial phase is not clear. Although the acidification and maturation phases overlapped during the lambic beer production processes of the present study, both phases could be distinguished based on the prevailing growth of $\mathrm{AAB}$ and LAB (both causing acidification) versus Pichia and Dekkera yeasts (marking the start of the maturation phase). It is hypothesized that the extent of separation of these two phases, as well as the distinction between $\mathrm{AAB}$ and $\mathrm{LAB}$ growth, depends on both the oxygen level and the ambient temperature. The oxygen level is of importance to promote $\mathrm{AAB}$ growth (Pothakos et al., 2016; De Roos et al., 2018). LAB need a temperature above $15^{\circ} \mathrm{C}$ to be able to grow during lambic beer production (De Keersmaecker, 1996). Except for AAB growth and the production of $\mathrm{AAB}$-related metabolites that took place mainly at the liquid/air interphase of the casks, no spatial differences occurred regarding yeast and $\mathrm{LAB}$ species diversity, substrate consumption, and metabolite production in the fermenting wort and maturing beer. As inoculation of the wort occurred before it was transferred into the casks, the microorganisms probably originated from the brewery air during cooling, contact with transfer tubings, or a combination of both. However, since previous research only recovered few yeasts or bacteria relevant for lambic beer fermentation from the coolship or brewery 
air, it was more likely that a meaningful inoculation occurred when the wort came into contact with other brewery surfaces (Bokulich et al., 2015; Spitaels et al., 2015). Tubings used for both matured lambic beer and fresh wort and other nonproduction equipment that encountered wort/matured lambic beer are often cleaned superficially and may thus harbor fermentation-related microorganisms. It has indeed been shown that substrate contact is the main actor in shaping the microbial community structures of different brewery surfaces (Bokulich et al., 2015). Recently, it has been shown that the wooden barrels can be an additional inoculation source too (De Roos et al., 2019).

During the lambic beer production processes studied, the prevalence of specific microbial species shifted as a function of time, which depended on a combination of both substrate consumption and metabolite production as well as environmental factors such as temperature and $\mathrm{pH}$. The enterobacteria present from the start of the fermentation disappeared quickly, due to increasing ethanol concentrations produced by the yeasts, decreasing $\mathrm{pH}$ values because of enterobacterial and $\mathrm{AAB}$ ( $A$. orientalis) acidification, and monosaccharide depletion by microbial growth in general (Priest and Stewart, 2006; De Roos et al., 2018). Besides enterobacteria and $\mathrm{AAB}$, Cellulosimicrobium species occurred during the enterobacterial phase (shown culture-independently only). However, since Cellulosimicrobium was never encountered before in lambic beer fermentations and yeast species generally dominate the main fermentation phase, it is likely that the bacterial DNA concentrations of these samples are very low and that the enzymes lyticase and Zymolyase used in the DNA extraction protocol may be the main source for these sequencing reads. Lyticase and Zymolyase are typically isolated from Cellulosimicrobium cellulans and this species has been found upon DNA extraction and high-throughput sequencing frequently (Ferrer, 2006; Verce et al., unpublished results).

Both $H$. uvarum and $S$. cerevisiae initiated the main fermentation phase. Fast consumption of the carbohydrate substrates available and the production of high ethanol concentrations led $S$. cerevisiae to take over from other yeast species, as this impaired growth of competing microorganisms (De Keersmaecker, 1996; Piškur et al., 2006; Rozpedowska et al., 2011; Spitaels et al., 2015). The transition from $H$. uvarum to $S$. cerevisiae is well described during spontaneous wine fermentations and is most likely caused by the production of certain S. cerevisiae metabolites, such as inhibitory ethanol and antimicrobial peptides (Branco et al., 2014; Wang et al., 2015). The slower decrease of other cycloheximide-resistant yeasts, such as Kazachstania species, indicated that such inhibitory effects are less pronounced, when compared with the Crabtree-negative H. uvarum (Hagman and Piškur, 2015). The occurrence of $H$. uvarum during the initial stages of the lambic beer fermentation process has been described before (Van Oevelen et al., 1977; Verachtert and Iserentant, 1995). In contrast, lambic beer fermentations do occur in the absence of H. uvarum (Spitaels et al., 2014, 2015). Hence, H. uvarum may not be necessary for lambic beer production, although it is known that $S$. cerevisiae is metabolically stimulated, under aerobic and anaerobic conditions, in co-cultivation with non-Saccharomyces yeasts (Curiel et al., 2017). Furthermore, Saccharomyces species are indispensable and are known to dominate the early stages of lambic beer fermentation, due to their fast carbohydrate consumption, ethanol production, accumulation and tolerance, and ability to ferment in the absence of oxygen (De Keersmaecker, 1996; Piškur et al., 2006; Spitaels et al., 2015). Probably due to an environmental temperature drop of the fermenting wort, the yeast species diversity shifted from the mesophilic $S$. cerevisiae to the cryotolerant $S$. kudriavzevii. The presence of $S$. kudriavzevii was not surprising, as this yeast species is associated with oak bark and can co-exist with S. cerevisiae and Saccharomyces paradoxus (Sampaioa and Goncalves, 2008). Moreover, S. cerevisiae-S. kudriavzevii hybrids are common in Belgian-style beers (González et al., 2008).

The main fermentation phase was characterized by a sequential consumption of mono-, di-, and trisaccharides and high production of yeast-associated metabolites, such as ethanol, succinic acid, and methyl-1-butanol. Exhaustion of maltose and maltotriose caused starvation of Saccharomyces species and was followed by the acidification phase. Also simultaneous and continuous breakdown of maltooligosaccharides occurred during this acidification phase. Although Dekkera species produce both intra- and extracellular $\alpha$-glucosidases, enzymes capable of dextrin degradation (Shanta Kumara et al., 1993), these species were only isolated from month 6 onward, whereas the continuous breakdown of maltooligosaccharides already started during the first month of fermentation. Moreover, it has been shown that the degradation of lower maltooligosaccharides happens faster than that of higher maltooligosaccharides when Dekkera species are involved (Shanta Kumara et al., 1993). Therefore, the breakdown of maltooligosaccharides during the acidification phase and even earlier was most likely due to the presence of yeast species at low counts $(<2.0 \log \mathrm{CFU} / \mathrm{ml})$ or as a result of cell lysis of yeast species present during the main fermentation phase, thereby releasing dextrin-degrading enzymes into the fermenting wort. Also acid hydrolysis cannot be ruled out, given the length of the lambic beer production process.

Acidification was partly due to AAB species, among which A. pasteurianus was most prevalent. The high production of acetic acid by $\mathrm{AAB}$ through oxidation of ethanol produced by the yeasts led to the formation of the ester ethyl acetate, probably due to a combination of enzymatic and chemical reactions (De Roos et al., 2018). Since no 2,3-butanedione or 2,3-butanediol occurred in the absence of LAB and presence of $\mathrm{AAB}$ during the first months of the acidification phase, the production of acetoin was due to the oxidation of lactic acid by AAB (Moens et al., 2014; De Roos et al., 2018). The preference of Acetobacteraceae to grow on ethanol and the continuous breakdown of maltooligosaccharides led to the elaboration of glucose, maltose, and maltotriose during the first part of the acidification phase, after which they were consumed by the LAB and yeasts. The later stages of the acidification phase allowed the growth of the LAB species $P$. damnosus, 
thanks to the increased environmental temperature. This led to lactic acid production and the formation of the ester ethyl lactate, which was again probably a combined effect of both chemical and enzymatic reactions. Whereas co-fermentation of glucose and malic acid took place by the LAB species present, citric acid was not metabolized. Indeed, it has been shown that for wine fermentations Pediococcus parvulus for example does not degrade citric acid during malolactic fermentation, as opposed to Oenococcus oeni (Davis et al., 1986). Although malic acid consumption has been reported in different yeast species as well ( $\mathrm{Su}$ et al., 2014), it is unlikely to be the case here, since $P$. membranifaciens was the only yeast species already detected in the fermenting wort in both casks before malic acid was consumed. Moreover, during the maturation phase, when $P$. membranifaciens co-occurred with Dekkera species, malic acid production rather than its consumption occurred. Up to now, malolactic fermentation was never reported during lambic beer production. Malolactic fermentation generally occurs in spontaneously fermented alcoholic beverages, such as wine (González-Arenzana et al., 2012, 2013a,b) and cider (Salih et al., 1988; Swaffield and Scott, 1995), and is carried out by many LAB species during fermentation of vegetables and fruits (Axelsson, 2004). It is performed in the commercial production of wine to convert tart L-malic acid into softer-tasting L-lactic acid (Fugelsang and Edwards, 2007) and can for the same reason be of importance during lambic beer production.

Biogenic amines were present at very low concentrations in the lambic brews of the present study, thanks to the limited growth of enterobacteria. In contrast, cheese and fermented sausage can contain much higher biogenic amine concentrations (EFSA Panel on Biological Hazards, 2011; Schirone et al., 2012; LatorreMoratalla et al., 2017). P. damnosus also contributed to biogenic amine (histamine) formation, although staying well below the concentrations that are acceptable for health $(<400 \mathrm{mg} / \mathrm{kg})$. As is the case in most fermented foods, histamine was present together with tyramine in the lambic beer production processes of the present study (Alvarez and Moreno-Arribas, 2014; del Rio et al., 2017). Although tyramine is known to be produced in beer by some Pediococcus species (Izquierdo-Pulido et al., 1997), it only increased after their prevalence during the acidification phase. It is possible that tyramine is produced by other LAB species present that were too low in abundance to be detected culturedependently or culture-independently. Another possibility is the release of tyramine into the fermenting wort and maturing beer due to autolysis of yeast cells (Blackwell et al., 1969; BonninJusserand et al., 2012).

Lactic acid bacteria and Dekkera species occurred together during the first months of the maturation phase. Co-occurrence of both microbial groups causes a more pronounced overattenuation (Shanta Kumara and Verachtert, 1991). Dekkera species and $P$. membranifaciens grew during the maturation phase, thereby consuming mainly carbohydrates to produce additional ethanol. The yeast species of the maturation phase were further responsible for pellicle formation, in turn decreasing the oxygen influx and therefore causing a decrease in AAB numbers (De Roos et al., 2018). Dekkera species are undesirable in wine but indispensable in lambic beer and Orval trappist productions. They have an important effect on the volatile aroma profile of lambic beers with, for example, the formation of ethyl acetate, ethyl lactate and phenethyl acetate, along with the hydrolysis of isoamyl acetate (Spaepen and Verachtert, 1982). They are also capable of producing 4-ethylguaiacol and 4ethylphenol, known as Brett flavor in lambic beer and Orval, under conditions of little residual carbohydrates during the maturation process (Chatonnet et al., 1995; Lentz and Harris, 2015). During the maturation phase, acetoin concentrations diminished, probably due to the use of acetoin, produced by AAB, as an alternative external electron acceptor by Dekkera species to reconcile their redox imbalance caused by their incapability to reoxidize $\mathrm{NADH}+\mathrm{H}^{+}$via the glycerol pathway under oxygen limitation (Steensels et al., 2015; De Roos et al., 2018).

\section{CONCLUSION}

The present study monitored the temporal and spatial distribution of microorganisms, substrates, and metabolites during lambic beer production processes, thereby unraveling reasons behind certain community dynamics such as the transition of Hanseniaspora and Kazachstania species to Saccharomyces species, and the community dynamics of AAB, $\mathrm{LAB}$, and yeasts during the acidification and maturation phases. Moreover, some new fermentation characteristics during lambic beer production were revealed, such as the occurrence of a malolactic fermentation by $P$. damnosus, the consumption of acetoin by Dekkera species, the simultaneous breakdown of maltooligosaccharides possibly by the yeasts, and the occurrence of a short enterobacterial phase despite manual wort acidification with lactic acid. In most lambic beer breweries, the latter is applied to enhance the microbial stability of the wort and to control biogenic amine production by enterobacteria. However, the data of the present study showed that, although the enterobacterial phase was shortened by this application, biogenic amines were still produced during the overnight cooling of the wort and the early stages of fermentation, albeit in very low concentrations, which do not have to be worried about. Moreover, also other microbial groups such as LAB contributed to the total biogenic amine contents. Therefore, wort acidification does not avoid biogenic amine production completely, although it contributes to the controllability of the biogenic amine production in particular and of the lambic beer production process in general by enhancing the microbial stability without hampering the fermentation and maturation process. This new knowledge of how the microbial communities are shaped throughout the lambic beer production process will contribute to its follow-up, which is mainly based on human sensory analysis nowadays.

\section{AUTHOR CONTRIBUTIONS}

JR contributed to the experimental work, the acquisition, processing, and interpretation of the data, and drafting of the 
manuscript. PV and LV interpreted the data, supervised the work, and reviewed and edited the manuscript.

\section{FUNDING}

This work was financially supported by the Research Council of the Vrije Universiteit Brussel (SRP7 and IOF342 projects), the Hercules Foundation (projects UABR09004 and UAB13002), and the KMO Portefeuille (projects

\section{REFERENCES}

Altschul, S. F., Gish, W., Miller, W., Myers, E. W., and Lipman, D. J. (1990). Basic local alignment search tool. J. Mol. Biol. 215, 403-410. doi: 10.1016/S00222836(05)80360-2

Alvarez, M. A., and Moreno-Arribas, V. (2014). The problem of biogenic amines in fermented foods and the use of potential biogenic amine-degrading microorganisms as a solution. Trends Food Sci. Technol. 39, 146-155. doi: 10.1016/j.tifs.2014.07.007

Axelsson, L. (2004). "Lactic acid bacteria: classification and physiology", in Lactic Acid Bacteria, Microbiological and Functional Aspects, eds S. Salminen, A. von Wright, and A. Ouwehand (New York, NY: Marcel Dekker), 1-66. doi: 10.1201/ 9780824752033.ch1

Blackwell, B., Mabitt, L. A., and Marley, E. (1969). Histamine and tyramine content of yeast products. J. Food Sci. 34, 47-51. doi: 10.1111/j.1365-2621.1969. tb14359.x

Bokulich, N. A., and Bamforth, C. W. (2013). The microbiology of malting and brewing. Microbiol. Mol. Biol. Rev. 77, 157-172. doi: 10.1128/MMBR.00 060-12

Bokulich, N. A., and Bamforth, C. W. (2017). Brewing Microbiology: Current Research, Omics and Microbial Ecology. Poole: Caister Academic Press, 332.

Bokulich, N. A., Bamforth, C. W., and Mills, D. A. (2012). Brewhouseresident microbiota are responsible for multi-stage fermentation of American coolship ale. PLoS One 7:e35507. doi: 10.1371/journal.pone.003 5507

Bokulich, N. A., Bergsveinson, J., Ziola, B., and Mills, D. A. (2015). Mapping microbial ecosystems and spoilage-gene flow in breweries highlights patterns of contamination and resistance. eLife 4:e04634. doi: 10.7554/eLife. 04634

Bokulich, N. A., and Mills, D. A. (2013). Improved selection of internal transcribed spacer-specific primers enables quantitative, ultra-high-throughput profiling of fungal communities. Appl. Environ. Microbiol. 79, 2519-2526. doi: 10.1128/ AEM.03870-12

Bonnin-Jusserand, M., Grandvalet, C., Rieu, A., Weidmann, S., and Alexandre, H. (2012). Tyrosine-containing peptides are precursors of tyramine produced by Lactobacillus plantarum strain IR BL0076 isolated from wine. BMC Microbiol. 12, 199-210. doi: 10.1186/1471-2180-12-199

Branco, P., Francisco, D., Chambon, C., Hébraud, M., Arneborg, N., Almeida, M. G., et al. (2014). Identification of novel GAPDH-derived antimicrobial peptides secreted by Saccharomyces cerevisiae and involved in wine microbial interactions. Appl. Microbiol. Biotechnol. 98, 843-853. doi: 10.1007/s00253-0135411-y

Briggs, D. E., Boulton, C., Brookes, P., and Stevens, R. (2004). Brewing Science and Practice. Cambridge: Woodhead Publishing Limited, 900. doi: 10.1201/ 9780203024195

Caporaso, J. G., Lauber, C. L., Walters, W. A., Berg-Lyons, D., Lozupone, C. A., Turnbaugh, P. J., et al. (2011). Global patterns of $16 \mathrm{~S}$ rRNA diversity at a depth of millions of sequences per sample. Proc. Natl. Acad. Sci. U.S.A. 108, 4516-4522. doi: 10.1073/pnas.1000080107

Chatonnet, P., Dubourdieu, D., and Boidron, J. N. (1995). The influence of Brettanomyces/Dekkera sp. yeasts and lactic acid bacteria on the ethylphenol content of red wines. Am. J. Enol. Viticult. 46, 463-468. doi: 10.1007/s00253013-5411-y
2014KMO084991, 2015KMO091056, 2016KMO149170, and 2017KMO112091, in collaboration with the brewery Oud Beersel).

\section{ACKNOWLEDGMENTS}

JR is the recipient of a Ph.D. fellowship of the Vrije Universiteit Brussel. We thank Wim Borremans for providing technical assistance with the substrate and metabolite analyses.

Curiel, J. A., Morales, P., Gonzalez, R., and Tronchoni, J. (2017). Different nonSaccharomyces yeast species stimulate nutrient consumption in S. cerevisiae mixed cultures. Front. Microbiol. 8:2121. doi: 10.3389/fmicb.2017.02121

Davis, C. R., Wibowo, D. J., Lee, T. H., and Fleet, G. H. (1986). Growth and metabolism of lactic acid bacteria during and after malolactic fermentation of wines at different pH. Appl. Environ. Microbiol. 51, 539-545.

De Bruyn, F., Zhang, S., Pothakos, V., Torres, J., Lambot, C., Moroni, A. V., et al. (2017). Exploring the impact of post-harvest processing on the microbiota and metabolite profiles during a case of green coffee bean production. Appl. Environ. Microbiol. 83:e002398-16.

De Keersmaecker, J. (1996). The mystery of lambic beer. Sci. Am. 275, 74-81. doi: 10.1038/scientificamerican0896-74

De Roos, J., and De Vuyst, L. (2018). Acetic acid bacteria in fermented foods and beverages. Curr. Opin. Biotechnol. 49, 115-119. doi: 10.1016/j.copbio.2017. 08.007

De Roos, J., Van der Veken, D., and De Vuyst, L. (2019). The interior surfaces of wooden barrels are an additional microbial inoculation source for lambic beer production. Appl. Environ. Microbiol. 85:e2226-18. doi: 10.1128/AEM.02 226-18

De Roos, J., Verce, M., Aerts, M., Vandamme, P., and De Vuyst, L. (2018). Temporal and spatial distribution of the acetic acid bacterium communities throughout the wooden casks used for the fermentation and maturation of lambic beer underlines their functional role. Appl. Environ. Microbiol. 84:e02846-17. doi: 10.1128/AEM.02846-17

del Rio, B., Redruello, B., Linares, D. M., Ladero, V., Fernandez, M., Martin, M. C., et al. (2017). The dietary biogenic amines tyramine and histamine show synergistic toxicity towards intestinal cells in culture. Food Chem. 218, 249-255. doi: 10.1016/j.foodchem.2016.09.046

Edgar, R. C., Haas, B. J., Clemente, J. C., Quince, C., and Knight, R. (2011). UCHIME improves sensitivity and speed of chimera detection. Bioinformatics 27, 2194-2200. doi: 10.1093/bioinformatics/btr381

EFSA Panel on Biological Hazards. (2011). Scientific opinion on scientific opinion on risk based control of biogenic amine formation in fermented foods. EFSA J. 9:2393. doi: 10.2903/j.efsa.2011.2393

Ferrer, P. (2006). Revisiting the Cellulosimicrobium cellulans yeast-lytic $\beta-1,3-$ glucanases toolbox: a review. Microb. Cell Fact. 5:10. doi: 10.1186/1475-28595- 10

Findley, K., Oh, J., Yang, J., Conlan, S., Deming, C., Meyer, J. A., et al. (2013). Topographic diversity of fungal and bacterial communities in human skin. Nature 498, 367-370. doi: 10.1038/nature12171

Fugelsang, K. C., and Edwards, C. G. (2007). "Microbial ecology during vinification," in Wine Microbiology, eds K. C. Fugelsang and C. G. Edwards (New York, NY: Springer), 82-101.

González-Arenzana, L., López, R., Santamaría, P., Tenorio, C., and López-Alfaro, I. (2012). Dynamics of indigenous lactic acid bacteria populations in wine fermentations from La Rioja (Spain) during three vintages. Microb. Ecol. 63, 12-19. doi: 10.1007/s00248-011-9911-y

González, S. S., Bario, E., and Querol, A. (2008). Molecular characterization of new natural hybrids of Saccharomyces cerevisiae and S. kudriavzevii in brewing. Appl. Microbiol. Biotechnol. 74, 2314-2320. doi: 10.1128/AEM.01867-07

González-Arenzana, L., López, R., Santamaría, P., and López-Alfaro, I. (2013a). Dynamics of lactic acid bacteria populations in Rioja wines by PCR-DGGE, comparison with culture-dependent methods. Appl. Microbiol. Biotechnol. 97, 6931-6941. doi: 10.1007/s00253-013-4974-y 
González-Arenzana, L., Santamaría, P., López, R., and López-Alfaro, I. (2013b). Indigenous lactic acid bacteria communities in alcoholic and malolactic fermentations of Tempranillo wines elaborated in ten wineries of La Rioja (Spain). Food Res. Int. 50, 438-445. doi: 10.1016/j.foodres.2012. 11.008

Gweon, H. S., Oliver, A., Taylor, J., Booth, T., Gibbs, M., Read, D. S., et al. (2015). PIPITS: an automated pipeline for analyses of fungal internal transcribed spacer sequences from the Illumina sequencing platform. Methods Ecol. Evol. 6, 973-980. doi: 10.1111/2041-210X.12399

Hagman, A., and Piškur, J. (2015). A study on the fundamental mechanism and the evolutionary driving forces behind aerobic fermentation in yeast. PLoS One 10:e0116942. doi: 10.1371/journal.pone.0116942

Howard, P. H. (2014). "Too big to ale? Globalization and consolidation in the beer industry," in The Geography of Beer: Regions, Environment, and Society, eds M. Patterson and N. Pullen (New York, NY: Springer), 155-165.

Izquierdo-Pulido, M., Carceller-Rosa, J.-M., Maine-Font, A., and VidalCaroue, M. C. (1997). Tyramine formation by Pediococcus spp. during beer fermentation. J. Food Protect. 60, 831-836. doi: 10.4315/0362-028X60.7.831

Izquierdo-Pulido, M., Font-Fábregas, J., and Vidal-Carou, C. (1995). Influence of Saccharomyces cerevisiae var. uvarum on histamine and tyramine formation during beer fermentation. Food Chem. 54, 51-54. doi: 10.1016/0308-8146(95) 92661-3

Izquierdo-Pulido, M., Hernández-Jover, T., Mariné-Font, A., and Vidal-Carou, M. C. (1996). Biogenic amines in European beers. J. Agric. Food Chem. 44, 3159-3163. doi: 10.1021/jf960155j

Kalac, P., and Krizek, M. (2003). A review of biogenic amines and polyamines in beer. J. Instit. Brew. 109, 123-128. doi: 10.1002/j.2050-0416.2003. tb00141.x

Latorre-Moratalla, M. L., Comas-Basté, O., Bover-Cid, S., and Vidal-Carou, M. C. (2017). Tyramine and histamine risk assessment related to consumption of dry fermented sausages by the Spanish population. Food Chem. Toxicol. 99, 78-85. doi: 10.1016/j.fct.2016.11.011

Laureys, D., and De Vuyst, L. (2014). Microbial species diversity, community dynamics, and metabolite kinetics of water kefir fermentation. Appl. Environ. Microbiol. 80, 2564-2572. doi: 10.1128/AEM.03978-13

Lentz, M., and Harris, C. (2015). Analysis of growth inhibition and metabolism of hydroxycinnamic acids by brewing and spoilage strains of Brettanomyces yeast. Foods 4, 581-593. doi: 10.3390/foods4040581

Martens, H., Dawoud, E., and Verachtert, H. (1991). Wort enterobacteria and other microbial populations involved during the 1st month of lambic fermentation. J. Instit. Brew. 97, 435-439. doi: 10.1002/j.2050-0416.1991. tb01082.x

Martens, H., Dawoud, E., and Verachtert, H. (1992). Synthesis of aroma compounds by wort enterobacteria during the 1st stage of lambic fermentation. J. Instit. Brew. 98, 421-425. doi: 10.1002/j.2050-0416.1992.tb0 1126.x

Martens, H., Iserentant, D., and Verachtert, H. (1997). Microbiological aspects of a mixed yeast-bacterial fermentation in the production of a special Belgian acidic ale. J. Instit. Brew. 103, 85-91. doi: 10.1002/j.2050-0416.1997.tb 00939.x

Martin, M. (2011). Cutadapt removes adapter sequences from high-throughput sequencing reads. EMBnet J. 17, 10-12. doi: 10.14806/ej.17.1.200

Moens, F., Lefeber, T., and De Vuyst, L. (2014). Oxidation of metabolites highlights the microbial interactions and role of Acetobacter pasteurianus during cocoa bean fermentation. Appl. Environ. Microbiol. 80, 1848-1857. doi: 10.1128/AEM. 03344-13

Oelofse, A., Pretorius, I. S., and du Toit, M. (2008). Significance of Brettanomyces and Dekkera during winemaking: a synoptic review. South Afr. J. Enol. Viticult. 29, 128-144.

Piškur, J., Rozpędowska, E., Polakova, S., Merico, A., and Compagno, C. (2006). How did Saccharomyces evolve to become a good brewer? Trends Genet. 22, 183-186. doi: 10.1016/j.tig.2006.02.002

Pothakos, V., Illeghems, K., Laureys, D., Spitaels, F., Vandamme, P., and De Vuyst, L. (2016). "Acetic acid bacteria in fermented food and beverage ecosystems," in Acetic Acid Bacteria: Ecology and Physiology, eds K. Matsushita, H. Toyama, N. Tonouchi, and A. Okamoto-Kainuma (Tokyo: Springer), $73-100$.
Priest, F. G., and Stewart, G. G. (2006). “Microbiology and microbiological control in the brewery," in Handbook of Brewing, 2nd Edn, eds F. G. Priest and G. G. Stewart (Boca Raton, FL: CRC Press), 607-629.

Rozpedowska, E., Hellborg, L., Ishchuk, O. P., Orhan, F., Galafassi, S., Merico, A., et al. (2011). Parallel evolution of the make-accumulate-consume strategy in Saccharomyces and Dekkera yeasts. Nat. Commun. 2:302. doi: 10.1038/ ncomms 1305

Salih, A., Drilleau, J., Cavin, F., Divies, C., and Bourgeois, C. (1988). A survey of microbiological aspects of cider making. J. Instit. Brew. 94, 5-8. doi: 10.1002/j. 2050-0416.1988.tb04545.x

Sampaioa, J. P., and Goncalves, P. (2008). Natural populations of Saccharomyces kudriavzevii in Portugal are associated with oak bark and are sympatric with S. cerevisiae and S. paradoxus. Appl. Environ. Microbiol. 77, 2144-2152. doi: 10.1128/AEM.02396-07

Schirone, M., Tofalo, R., Visciano, P., Corsetti, A., and Suzzi, G. (2012). Biogenic amines in Italian Pecorino cheese. Front. Microbiol. 3:171. doi: 10.3389/fmicb. 2012.00171

Schloss, P. D., Westcott, S. L., Ryabin, T., Hall, J. R., Hartmann, M., Hollister, E. B., et al. (2009). Introducing Mothur: open-source, platform-independent, community-supported software for describing and comparing microbial communities. Appl. Environ. Microbiol. 75, 7537-7541. doi: 10.1128/AEM. 01541-09

Shanta Kumara, H. M. C., Decort, S., and Verachtert, H. (1993). Localization and characterization of $\alpha$-glucosidase activity in Brettanomyces lambicus. Appl. Environ. Microbiol. 59, 2352-2358.

Shanta Kumara, H. M. C., and Verachtert, H. (1991). Identification of lambic superattenuating microorganisms by the use of selective antibiotics. J. Instit. Brew. 97, 181-185. doi: 10.1002/j.2050-0416.1991.tb01064.x

Snauwaert, I., Roels, S. P., Van Nieuwerburg, F., Van Landschoot, A., De Vuyst, L., and Vandamme, P. (2016). Microbial diversity and metabolite composition of Belgian red-brown acidic ales. Int. J. Food Microbiol. 221, 1-11. doi: 10.1016/j. ijfoodmicro.2015.12.009

Spaepen, M., Van Oevelen, D., and Verachtert, H. (1978). Fatty acids and esters produced during the spontaneous fermentation of lambic and gueuze. J. Instit. Brew. 84, 278-282. doi: 10.1002/j.2050-0416.1978.tb03888.x

Spaepen, M., Van Oevelen, D., and Verachtert, H. (1979). Higher fatty acid (HFA) and HFA-ester content of spontaneously fermented Belgian beers and evaluation of their analytical determination. Brauwissenschaft 32, S1-S6.

Spaepen, M., and Verachtert, H. (1982). Esterase activity in the genus Brettanomyces. J. Instit. Brew. 88, 11-17. doi: 10.1002/j.2050-0416.1982. tb04061.x

Spano, G., Russo, P., Lonvaud-Funel, A., Lucas, P., Alexandre, H., Grandvalet, C., et al. (2010). Biogenic amines in fermented foods. Eur. J. Clin. Nutr. 64, S95-S100. doi: 10.1038/ejen.2010.218

Spitaels, F., Wieme, A. D., Janssens, M., Aerts, M., Daniel, H.-M., Van Landschoot, A., et al. (2014). The microbial diversity of traditional spontaneously fermented lambic beer. PLoS One 9:e95384. doi: 10.1371/journal. pone.0095384

Spitaels, F., Wieme, A. D., Janssens, M., Aerts, M., Van Landschoot, A., De Vuyst, L., et al. (2015). The microbial diversity of an industrially produced lambic beer shares members of a traditionally produced one and reveals a core microbiota for lambic beer fermentation. Food Microbiol. 49, 23-32. doi: 10.1016/j.fm.2015.01.008

Spitaels, F., Wieme, A. D., Snauwaert, I., De Vuyst, L., and Vandamme, P. (2017). "Microbial ecology of traditional beer fermentations," in Brewing Microbiology: Current Research, Omics and Microbial Ecology, eds N. Bokulich and C. Bamforth (Poole: Caister Academic Press), 179-196.

Steensels, J., Daenen, L., Malcorps, P., Derdelinckx, G., Verachtert, H., and Verstrepen, K. J. (2015). Brettanomyces yeasts - From spoilage organisms to valuable contributors to industrial fermentations. Int. J. Food Microbiol. 206, 24-38. doi: 10.1016/j.ijfoodmicro.2015.04.005

Su, J., Wang, T., Wang, Y., Li, Y.-Y., and Li, H. (2014). The use of lactic acidproducing, malic acid-producing, or malic acid-degrading yeast strains for acidity adjustment in the wine industry. Appl. Microbiol. Biotechnol. 98, 23952413. doi: 10.1007/s00253-014-5508-y

Swaffield, C. H., and Scott, J. A. (1995). Existence and development of natural microbial populations in wooden storage vats used for alcoholic cider maturation. J. Am. Soc. Brew. Chem. 53, 117-120. doi: 10.1094/ASBCJ-53-0117 
Van Oevelen, D., L'Escaille, F., and Verachtert, H. (1976). Synthesis of aroma components during the spontaneous fermentation of lambic and gueuze. J. Instit. Brew. 82, 322-326. doi: 10.1002/j.2050-0416.1975.tb06 953.x

Van Oevelen, D., Spaepen, M., Timmermans, P., and Verachtert, H. (1977). Microbiological aspects of spontaneous wort fermentation in the production of lambic and gueuze. . J. Instit. Brew. 83, 356-360. doi: 10.1002/j.2050-0416. 1977.tb03825.x

Verachtert, H. (1983). De spontane geuzegisting - La fermentation spontanée de la geuze. Cerevisia Belgian J. Brew. Biotechnol. 8, 41-48.

Verachtert, H., and Dawoud, E. (1984). Microbiology of lambic-type beers. J. Appl. Bacteriol. 57, R11-R12.

Verachtert, H., Dawoud, E., and Kumara, H. M. C. S. (1989). Interactions between Enterobacteriaceae and Saccharomyces cerevisiae during wort fermentation. Yeast 5, 67-72.

Verachtert, H., and Derdelinckx, G. (2005). Acidic beers: enjoyable reminiscences of the past. Cerevisia Belgian J. Brew. Biotechnol. 30, 38-47.

Verachtert, H., and Iserentant, D. (1995). Properties of Belgian acid beers and their microflora. Part I. The production of gueuze and related refreshing acid beers. Cerevisia Belgian J. Brew. Biotechnol. 20, 37-41.
Vermote, L., Verce, M., De Vuyst, L., and Weckx, S. (2018). Amplicon and shotgun metagenomic sequencing indicates that microbial ecosystems present in cheese brines reflect environmental inoculation during the cheese production process. Int. Dairy J. 87, 44-53. doi: 10.1016/j.idairyj.2018.07.010

Wang, C., Albert, M., and Esteve-Zarzoso, B. (2015). Interaction between Hanseniaspora uvarum and Saccharomyces cerevisiae during alcoholic fermentation. Int. J. Food Microbiol. 206, 67-74. doi: 10.1016/j.ijfoodmicro. 2015.04.022

Conflict of Interest Statement: The authors declare that the research was conducted in the absence of any commercial or financial relationships that could be construed as a potential conflict of interest.

Copyright (c) 2018 De Roos, Vandamme and De Vuyst. This is an open-access article distributed under the terms of the Creative Commons Attribution License (CC BY). The use, distribution or reproduction in other forums is permitted, provided the original author(s) and the copyright owner(s) are credited and that the original publication in this journal is cited, in accordance with accepted academic practice. No use, distribution or reproduction is permitted which does not comply with these terms. 